\title{
Orbital structure and mass distribution in elliptical galaxies
}

\author{
Andi Kronawitter ${ }^{1}$, R.P. Saglia ${ }^{2, \star}$, Ortwin Gerhard ${ }^{1}$, and Ralf Bender ${ }^{2}$ \\ 1 Astronomisches Institut, Universität Basel, Venusstrasse 7, CH-4102 Binningen, Switzerland \\ 2 Institut für Astronomie und Astrophysik, Scheinerstraße 1, D-81679 Munich, Germany
}

Received December 23, 1999; accepted February 15, 2000

\begin{abstract}
We report on a homogeneous dynamical analysis of a sample of 21 round (17 E0/E1, 4 E2) elliptical galaxies. We present new kinematic data for eight of these galaxies and new photometry for one object. The remaining kinematic and photometric data and the required distance information are taken from the literature. The analysis uses non-parametric spherical models and takes into account line profile information as well as velocity dispersions. We present model fits to the kinematic data and the derived radial profiles of orbital anisotropy and $B$-band mass-to-light ratio, including confidence intervals. The circular velocity curves resulting from our model fits are all consistent with being flat outside $R \approx 0.3 R_{\mathrm{e}}$. Generally, the $M / L$ ratio profiles show an outward increase, although models based on luminous matter are ruled out at $95 \%$ confidence only for three galaxies (NGC 2434, NGC 7507, NGC 7626). For NGC 1399, NGC 4472, NGC 4486, and NGC 4636, where $\mathrm{X}$-ray observations are available, the mass profiles of the best fit models match the ones derived from the X-ray analysis. The best models for most galaxies are isotropic to slightly radially anisotropic, with typical $\beta \lesssim 0.3$, in a few cases $\beta \lesssim 0.5$ at $R_{\mathrm{e}} / 2$. We discuss the generally small effects of flattening along the line-of-sight (the expected $<c / a>=0.79$ for this sample of luminous ellipticals) and of small embedded disks. Our results suggest that elliptical galaxies have surprisingly uniform dynamical properties.
\end{abstract}

Key words: galaxies: elliptical and lenticular, $\mathrm{cD}$ galaxies: fundamental parameters, kinematics and dynamics, photometry, structure

Send offprint requests to: A. Kronawitter

e-mail: krona@astro.unibas.ch

* Visiting astronomer at the German-Spanish Astronomical Center, Calar Alto, operated by the Max-Planck-Institut für Astronomie, Heidelberg jointly with the Spanish National Commission for Astronomy.

\section{Introduction}

Much less is known about the mass distributions of elliptical galaxies than for spirals. Ellipticals are hot stellar systems, and their circular velocity curves cannot be directly measured, but must be indirectly inferred from fitting dynamical models to the kinematic data. Has the luminous matter segregated dissipatively in the halo potential? Is there a "conspiracy" between luminous and dark matter to produce a flat rotation curve, like in spiral galaxies? How do the mass-to-light ratio, the slope of the circular velocity curve, or the orbital anisotropy scale with luminosity? The purpose of this paper is to construct a sample of elliptical galaxies for which these quantities are wellenough known to address these questions.

Evidence for dark matter in ellipticals comes from X-ray data on their hot gas atmospheres (e.g., Matsushita et al. 1998; Loewenstein \& White 1999), from a few cases with cold gas rings (Bertola et al. 1993), from gravitational lensing analyses (Keeton et al. 1998) and from stellardynamical work. Saglia et al. (1992) studied the velocity dispersion profiles of a sample of 10 bright ellipticals using anisotropic two-component models, finding that the amount of dark matter inside the half-luminosity radius is of the order of the luminous mass. Similar results were obtained modeling the extended profiles of Saglia et al. (1993) and Bertin et al. (1994), but these authors also showed that some of their galaxies could be modelled with constant mass-to-light ratio when more complicated, tangentially anisotropic models were employed.

Van der Marel (1991) constructed axisymmetric two-integral models for a sample of 37 bright ellipticals. He found that these models predicted too much motion on the major axis and interpreted this as evidence for anisotropy in the form $\sigma_{\mathrm{r}}>\sigma_{\theta}$. From the model fits he also derived mass-to-light ratios and concluded that these rise approximately as $\propto L^{0.35}$. These results were based on velocity dispersion and rotation measurements. Both modelling approaches described above are subject to some uncertainty, because of the well-known degeneracy 
Table 1. Setups of the spectroscopic runs. Column 1 gives the run number, Col. 2 the date, Col. 3 the telescope used, Col. 4 the instrument, Col. 5 the detector, Col. 6 the wavelength range, Col. 7 the scale in arcsec/pixel, Col. 8 the slit width in arcsec, Col. 9 the instrumental resolution in $\mathrm{km} \mathrm{s}^{-1}$

\begin{tabular}{|c|c|c|c|c|c|c|c|c|}
\hline Run & Date & Telescope $^{1}$ & Instrument $^{2}$ & Detector & $\begin{array}{c}\lambda-\text { range } \\
{[\AA]}\end{array}$ & $\begin{array}{c}\text { Scale } \\
{[" / \text { pix }]}\end{array}$ & $\begin{array}{l}\text { Slit- } \\
\text { width }\end{array}$ & {$\left[\begin{array}{c}\sigma_{\text {inst }} \\
{\left[\mathrm{km} \mathrm{s}^{-1}\right]}\end{array}\right.$} \\
\hline 1 & 31.10-2.11.1992 & NTT & EmmiRed & Tek $1024 \times 102419 \mu$ & $4890-5430$ & 0. & $1^{\prime \prime}$ & 70 \\
\hline 2 & 4-7.6.1994 & NTT Remote & EmmiRed & Tek $2048 \times 204824 \mu$ & $4826-5474$ & $0^{\prime \prime} 268$ & 3. & 85 \\
\hline 3 & 26-30.9.1994 & $3.5 \mathrm{CA}$ & TwinRed & Tek $1024 \times 102424 \mu$ & $4760-5640$ & $0 . .896$ & $33^{\prime \prime} 6$ & 85 \\
\hline 4 & 6-8.6.1994 & NTT Remote & EmmiRed & Tek $2048 \times 204824 \mu$ & $4826-5474$ & $0^{\prime \prime} 268$ & 3. & 85 \\
\hline 5 & 26-29.8.1995 & $3.5 \mathrm{CA}$ & TwinRed & Tek $1024 \times 102424 \mu$ & $4760-5640$ & $0 . .896$ & $3{ }^{\prime \prime} 6$ & 85 \\
\hline 6 & 10-12.3.1996 & NTT Remote & EmmiRed & Tek $2048 \times 204824 \mu$ & $4826-5474$ & 0.268 & 3. & 85 \\
\hline
\end{tabular}

(1) NTT: ESO 3.5 m New Technology Telescope; 3.5 CA: 3.5 Calar Alto Telescope.

(2) EmmiRed: Red Arm of EMMI; TwinRed: Red arm of the twin spectrograph.

between anisotropy and mass distribution, given only rotation and velocity dispersion data.

In the meantime, both the quality of the available kinematic data and of the dynamical modelling has improved greatly. Line profile shape measurements are now available for many ellipticals (e.g., Bender et al. 1994), and in a number of cases these measurements now extend to sufficiently large radii $\left(\sim 1-2 R_{\mathrm{e}}\right)$ that they begin to constrain the halo mass distribution (e.g., Carollo et al. 1995). Theoretical work has shown that such absorption line profile data contain sufficient information to extract constraints on both anisotropy and mass distribution (Gerhard 1993; Merritt 1993). In several recent studies, modern data together with sophisticated modelling methods were used to determine improved mass-to-light ratios and to estimate $M(r)$ and the anisotropy structure in individual elliptical galaxies (Rix et al. 1997; Gerhard et al. 1998; Emsellem et al. 1999; Cretton \& van den Bosch 1999; Saglia et al. 2000; Matthias \& Gerhard 1999; Gebhardt et al. 2000).

The goal of this paper is to extend this work to a sample of elliptical galaxies. We have selected galaxies that (i) cover as wide a range in total magnitudes and $R_{\mathrm{e}}$ as possible, in order to probe different regions of the Fundamental Plane, and (ii) are nearly round (E0E2), with small rotational velocities, so as to allow us to use spherical, non-rotating models in analyzing the data. We have obtained new kinematic data to $\sim 1-2 R_{\mathrm{e}}$ for eight galaxies. In addition, we have used extended kinematic data for NGC 1399 from Saglia et al. (2000), for NGC 2434 from Carollo \& Danziger (1994), for NGC 3379 from Statler \& Smecker-Hane (1999), and for NGC 6703 from Gerhard et al. (1998). To these we have added a second sample of ellipticals with kinematic data to smaller radii, from Bender et al. (1994, hereafter BSG94), in order to extend the total sample to less luminous galaxies and to increase the sample size. Dynamical models for all of these are shown in this paper. Based on the results of these models we will be able to investigate the family properties of ellipticals regarding mass-to-light ratios and anisotropies; this will be described in a forthcoming paper.

This paper is organised as follows: New spectroscopic observations are presented in Sect. 2. In Sect. 3 we describe the photometric data used for the modelling, as well as the distances employed in calculating masses and luminosities. Our modelling technique, which follows Gerhard et al. (1998, hereafter G+98) and Saglia et al. (2000, hereafter $\mathrm{S}+2000)$, is briefly discussed in Sect. 4. Results are given in Sect. 5, and in Sect. 6 we present our conclusions.

\section{Spectroscopic observations}

The spectroscopic data were collected during six runs of observations, between 1992 and 1996, using the ESO NTT in La Silla (the first run in visitor mode and the other three in remote observing mode from Garching) and the $3.5 \mathrm{~m}$ telescope in Calar Alto. Table 1 summarizes the instruments and detectors used and their setups. Table 2 gives the log of the observations. To maximize the signal at large radii, large slit widths were used. These rather than seeing set the spatial resolution of the observations in all cases except run \#1.

The standard reduction steps (bias subtraction, flat fielding, cosmic ray removal, logarithmic wavelength calibration, sky subtraction) were performed under MIDAS. A sky subtraction better than 1 per cent was achieved. The analysis of the data was carried out using the FCQ method (Bender 1990) following BSG94, G+98 and $\mathrm{S}+2000$. The spectra were rebinned along the spatial direction to obtain a nearly constant signal-to-noise ratio larger than 50 per resolution element. The effects of the continuum 
Table 2. Log of the spectroscopic observations. Column 1 gives the galaxy name, Col. 2 the run number (see Table 1), Col. 3 the slit position angle in degrees, Col. 4 the position across the galaxy (see notes), Col. 5 the shift from the center (see text), Col. 6 the exposure time, Col. 7 the number of collected frames

\begin{tabular}{lrrcccc}
\hline Galaxy & Run & $\begin{array}{r}\text { PA } \\
{\left[{ }^{\circ}\right]}\end{array}$ & Position $^{1}$ & $\begin{array}{c}\text { Shift } \\
{\left[{ }^{\prime \prime}\right]}\end{array}$ & $\begin{array}{c}\text { Exp.Time } \\
{[\text { hours }]}\end{array}$ & $\begin{array}{c}\text { Number } \\
\text { of Frames }\end{array}$ \\
\hline \multirow{2}{*}{ NGC 315 } & 2 & 44 & MJ & - & 0.71 & 1 \\
& 5 & 135 & pMN & 58 & 6.72 & 5 \\
NGC 3379 & 6 & 80 & MJ & - & 1.5 & 1 \\
& 6 & 170 & pMN & 35 & 12 & 8 \\
NGC 4374 & 6 & 126 & MJ & - & 1.5 & 1 \\
& 6 & 36 & pMN & 17 & 1.8 & 2 \\
NGC 5846 & 6 & 36 & pMN & 55 & 3.3 & 2 \\
& 2 & 70 & MJ & - & 2.5 & 2 \\
NGC 7145 & 2 & 160 & pMN & 80 & 7.5 & 5 \\
& 2 & 270 & pMN & - & 1.5 & 1 \\
NGC 7192 & 4 & 10 & MJ & - & 14.3 & 12 \\
NGC 7507 & 1 & 72 & D & - & 1.5 & 1 \\
& $1^{2}$ & 18 & pMN & 48 & 5 & 2 \\
NGC 7626 & 3 & 15 & MJ & - & 1.5 & 5 \\
& 5 & 105 & pMN & 57 & 9.4 & 7 \\
& & & & & & 1.5 \\
\hline
\end{tabular}

(1) MJ: major axis; pMN: parallel to the minor axis; D: diagonal. (2) Slit width $1.5^{\prime \prime}$.

fitting and instrumental resolution were extensively tested by Monte Carlo simulations. The residual systematic effects on the values of the $h_{3}$ and $h_{4}$ profiles are less than 0.01 , and less than $1.5 \%$ in $\sigma$. In general, in the inner parts of the galaxies the errorbars (which reflect the random errors and do not take into account systematic effects such as template mismatching or the presence of dust and faint emission, see below) are small, in the range $3-7 \mathrm{~km} \mathrm{~s}^{-1}$ for the recessional velocities and velocity dispersion, and $0.006-0.02$ for the $h_{3}$ and $h_{4}$ coefficients. The errors are larger in the outer parts, where the signal-to-noise ratio can be lower. They are calibrated with simulations and are determined to better than 20\%. Figures 1 and 2 present the results. Figures 3-8 compare the data to the literature. We discuss individual galaxies here below. Note that NGC 315, NGC 7507 and NGC 7145 appear to have noisier data than the rest, despite the long integration times (see Table 2). NGC 315 is the most distant galaxy of the sample, NGC 7507 has been observed with a tighter slit and NGC 7145 is the intrinsically faintest galaxy of the sample (see Table 3).

\section{1. $N G C 315$}

The spectra in the inner 2 arcsec show variable $\mathrm{H} \beta$ emission, which is probably responsible for the jumps observed in the $v$ and $\sigma$ values. The two most distant datapoints have rather poor signal-to-noise ratio. The related values of $h_{3}$ and $h_{4}$ are very uncertain; only the values of $\sigma$ and $v$ are given. The comparison with the old data of
Schechter \& Gunn (1979) and the velocity profile of Davies \& Birkinschaw (1988) (Fig. 3) is good within the rather large errors of the literature data.

\section{2. $N G C 3379$}

The data taken along the major axis of the galaxy follow closely the results of Statler \& Smecker-Hane (1999) (Fig. 4). The data along the shifted direction reach 100 arcsec from the center and show a declining (cylindrical) velocity, with negative values at 100 arcsec confirmed in the 8 separate frames.

The velocity dispersions and $h_{4}$ values along the major axis given in BSG94 (not shown here) appear slightly larger. The $h_{3}$ values and velocities agree well.

\section{3. $N G C 4374$}

The galaxy shows OIII emission in the inner 3 arcsec, where some differences between the two sides of the galaxy in the (folded) velocity, $h_{3}$ and $\sigma$ appear larger than the errorbars. No rotation is detected along the major axis; there is a hint of a $10 \mathrm{~km} \mathrm{~s}^{-1}$ (cylindrical) rotation as measured parallel to the minor axis (the 17 arcsec shift). Apart from the inner 3 arcsec (where emission is seen) there is no template mismatching. The last datapoint has low $\mathrm{S} / \mathrm{N}$ and $h_{4}$ might not be reliable. The antisymmetric term $h_{3}$ is approximately zero everywhere; the $h_{4}$ is small and slightly positive.

The comparison with the $v$ and $\sigma$ profile of Davies and Birkinshaw (1988) is satisfactory (Fig. 3). Their $\sigma$ data tend to be systematically higher than ours (at most $20 \mathrm{~km} \mathrm{~s}^{-1}$ at $R=10 \operatorname{arcsec}$ ). This might be due to their bad spectral resolution and/or differences in the slit aperture. Carollo et al. (1993) compare their data (affected by rather large errors) with Davies \& Birkinshaw (1988), finding agreement.

\section{4. $N G C 5846$}

The galaxy shows a slowly declining velocity dispersion, with no rotation and small $h_{3}$ and $h_{4}$ values.

Comparisons are given for the $v$ and $\sigma$ profiles to the data of Franx et al. (1989) and BSG94, where reasonable agreement is obtained (Fig. 5). The same is achieved for the data of Carollo et al. (1993), which have large errors and are not shown here. Sembach \& Tonry (1996) obtained a radially extended major axis kinematic profile with a pioneering (hardware) method to perform sky subtraction. The authors themselves note that their values are $6-7 \%$ higher than previous observations. Somewhat higher bias is observed here. The $h_{3}$ and $h_{4}$ data given by BSG94 are compatible with the present dataset, within their large errors. 

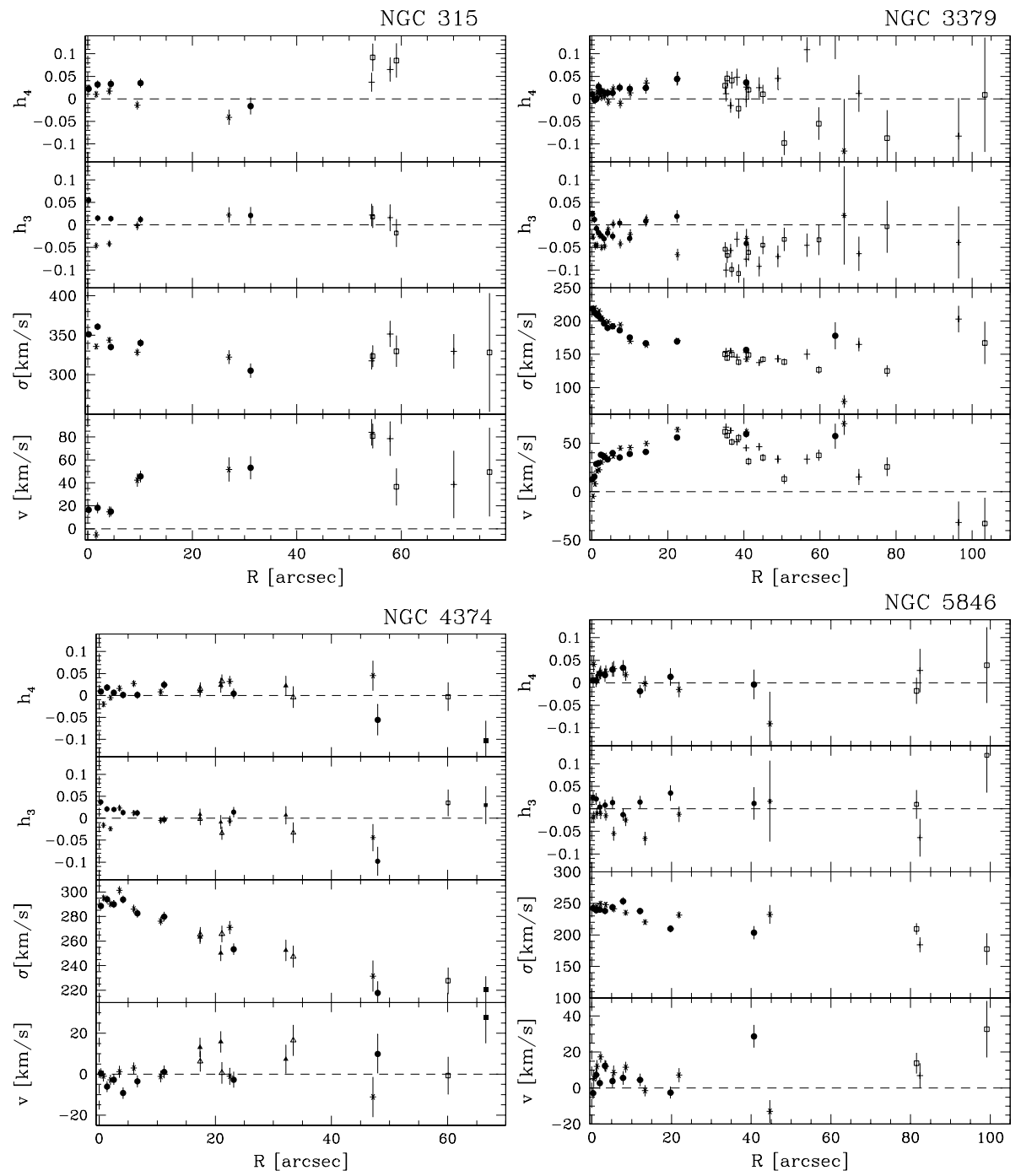

Fig. 1. The kinematics of NGC 315, NGC 3379, NGC 4374, NGC 5846. From bottom to top: the folded mean velocity, velocity dispersion, $h_{3}$ and $h_{4}$ profiles. Stars and filled circles refer to the two sides of the galaxy and the major axis spectrum. Crosses and open squares refer to the two sides of the galaxy and the spectra taken parallel to the minor axis and shifted 58 (NGC 315), 35 (NGC 3379), 55 (NGC 4374) and 80 (NGC 5846) arcsec from the center. Open and filled triangles refer to the two sides of NGC 4374 and the spectra taken parallel to the minor axis and shifted 17 arcsec from the center

\section{5. $N G C 7145$}

The galaxy has a rather low velocity dispersion, at the limit of the instrumental resolution $\left(\sigma \approx 120 \mathrm{~km} \mathrm{~s}^{-1}\right.$, $\left.\sigma_{\text {inst }}=85 \mathrm{~km} \mathrm{~s}^{-1}\right)$. Simulations show that there might be still some systematic effects at the $3 \%$ level in $\sigma$ and $0.01-0.02$ in $h_{4}$. The velocity data parallel to the minor axis are mirrored antisymmetrically with respect to the major axis. The velocity dispersions measured by Franx et al. (1989) are consistent within the errors. Their velocity measured along $\mathrm{PA}=132^{0}$ matches our value detected parallel to the minor axis (Fig. 6).

\section{6. $N G C$ 7192}

The galaxy has some OIII emission and is detected in $\mathrm{H} \alpha$ (Macchetto et al. 1996). The scatter in the velocities is probably due to this component. The velocity dispersion profile agrees with the central value $\left(185 \mathrm{~km} \mathrm{~s}^{-1}\right)$ of Faber et al. (1989). The kinematic data of Carollo \& Danziger (1994) are however completely off, being 50 to $70 \mathrm{~km} \mathrm{~s}^{-1}$ larger (Fig. 6). The fact that their major and minor axis data do not agree suggests that this dataset is not reliable.

\section{7. $N G C 7507$}

The galaxy shows a slowly declining velocity dispersion profile, with low rotation, small $h_{3}$ and slightly positive $h_{4}$ values. The systematic differences with the velocity dispersion profiles of Franx et al. (1989) and Bertin et al. (1994, Fig. 7) might be partly due to the 1 arcsec slit used here. 

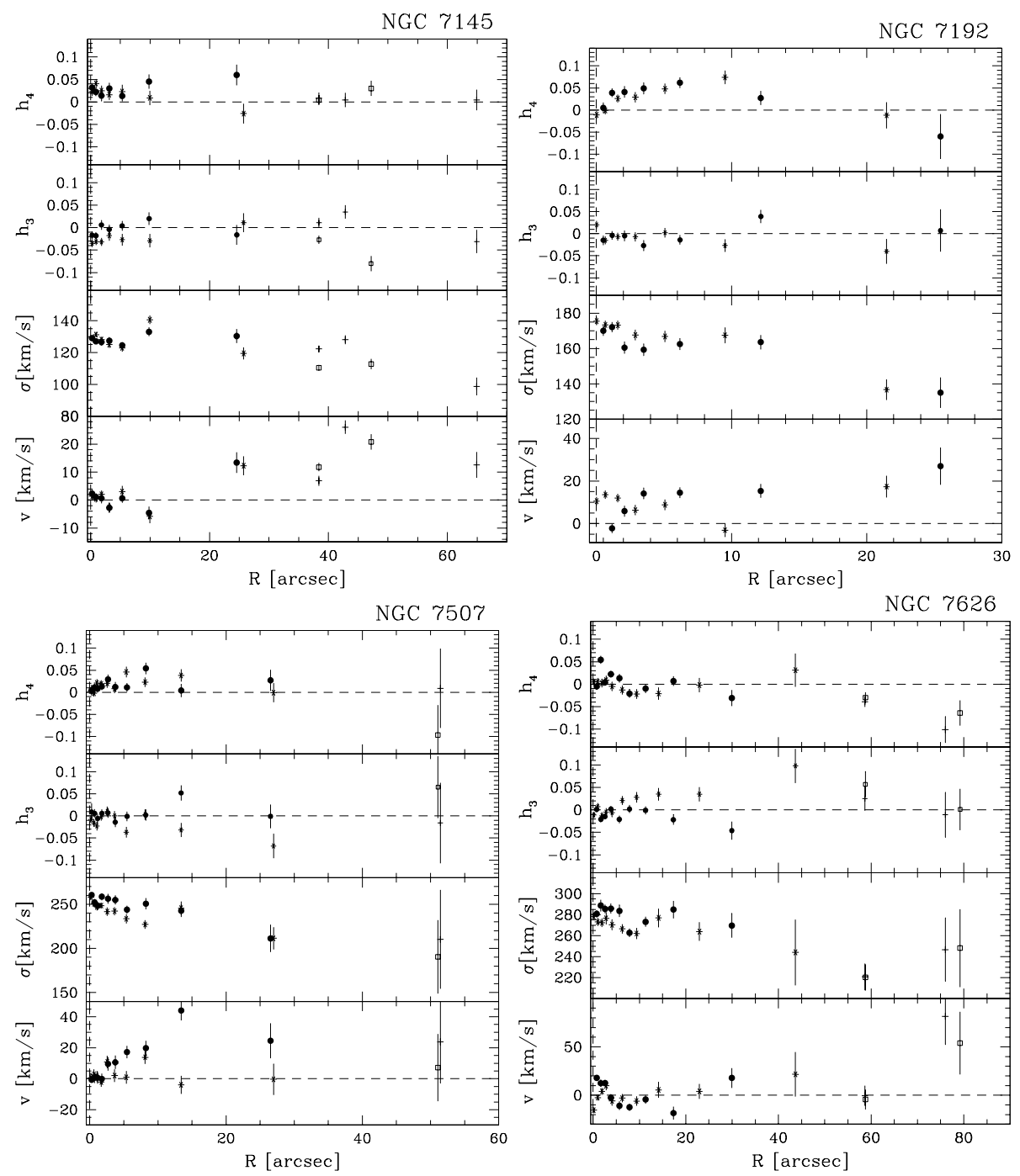

Fig. 2. The kinematics of NGC 7145, NGC 7192, NGC 7507, NGC 7626. From bottom to top: the folded mean velocity, velocity dispersion, $h_{3}$ and $h_{4}$ profiles. Stars and filled circles refer to the two sides of the galaxy and the major axis spectrum. Crosses and open squares refer to the two sides of the galaxy and the spectra taken parallel to the minor axis and shifted 38 (NGC 7145), 48 (NGC 7507) and 57 (NGC 7626) arcsec from the center

\section{8. $N G C 7626$}

As discussed in Balcells \& Carter (1993), the inner region of this galaxy is not relaxed. The differences between its two sides are real and reflect the complex kinematics. Some template mismatching is still present at $R \approx 10$ 30 arcsec.

The comparison with the velocity and velocity dispersion profiles of Balcells \& Carter (1993) is good within the errors; the $\sigma$ data of Jedrzejewski \& Schechter (1989) are systematically on the lower side.

\section{Photometry and distances}

Table 3 gives characteristic photometric quantities and distances for all galaxies in our combined sample. Since the luminosity distribution is needed to calculate the mass-tolight ratio, we check the calibration of the adopted surface brightness profiles as follows. We take photoelectric aperture measurements of Burstein et al. (1987), correct them for extinction and convert to the distances listed in Table 3 . The resulting aperture magnitudes are compared to the values obtained by integrating our (CCD-) SB profiles (converted to $B$-band when necessary) out to the radius of the aperture. Only two galaxies appear not correctly calibrated: NGC 1399 and NGC 7507. For these two cases we correct the final mass-to-light ratios to the aperture magnitude system given by Burstein et al. (1987).

Distances to elliptical galaxies are still uncertain and the scatter between the values based on different methods is considerable. Therefore, we have adopted the uniform set of group distances based on the Dn-sigma distance estimator from Faber et al. (1989; Col. $R$ of 

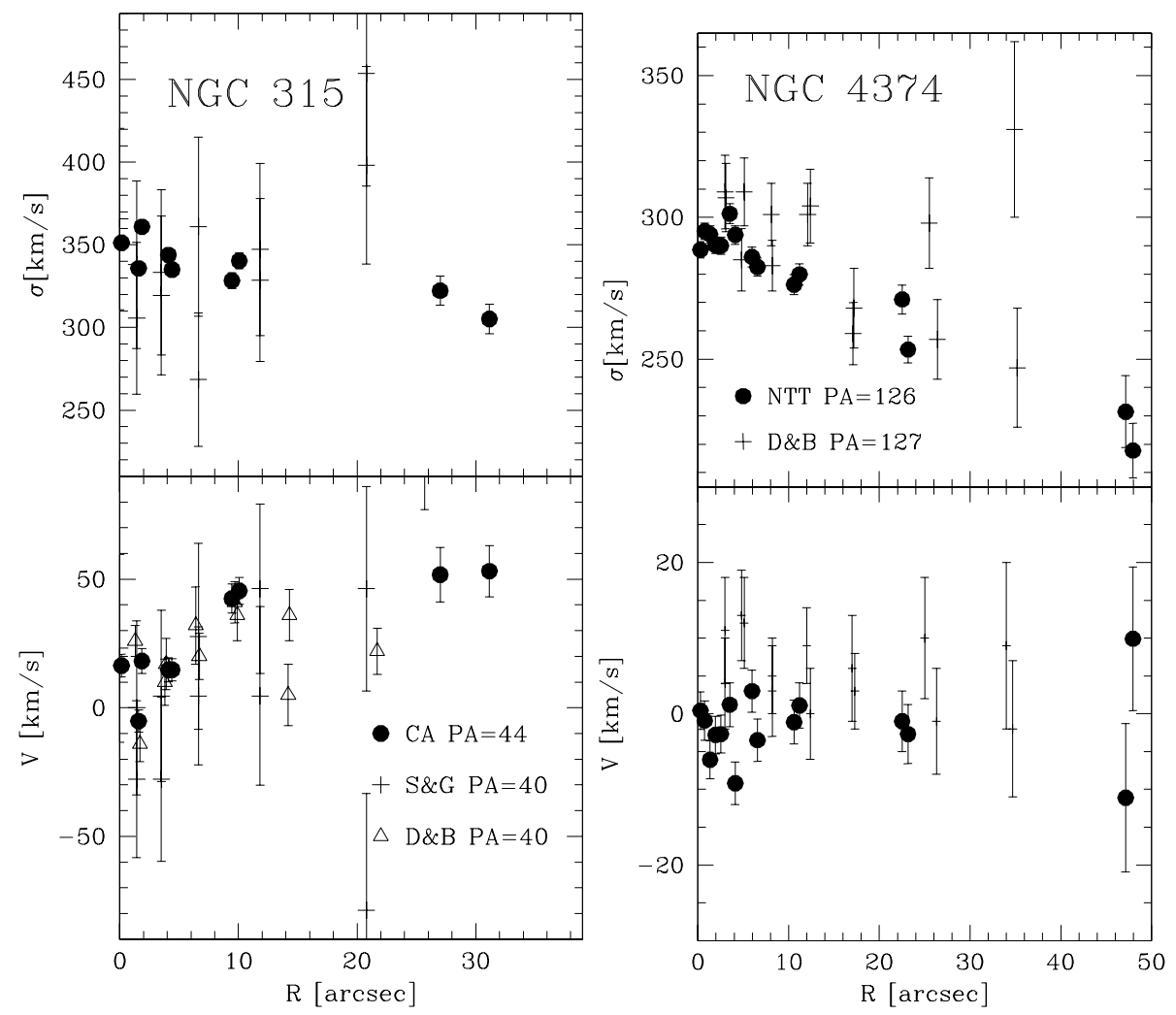

Fig. 3. Left: comparison with the velocity dispersion (top) and rotation velocities (bottom) of Schechter \& Gunn (1979, crosses) and Davies \& Birkinshaw (1988, only velocities, open triangles) for NGC 315. Right: comparison with the velocity dispersion (top) and rotation velocities (bottom) of Davies \& Birkinshaw (1988, crosses) for NGC 4374. The data of Carollo et al. (1993) have large error bars, within which they agree with our profiles

their Table 3), converting to Mpc with a Hubble constant of $H_{0}=65 \mathrm{~km} \mathrm{~s}^{-1} / \mathrm{Mpc}$. All $M / L$ values listed below and shown in Figs. 20 and 21 are transformed to these "standard distances" given in Table 3.

\section{1. $N G C 315$}

The SB-profile of Peletier et al. (1990) extends from 3.8 arcsec out to 91.8 arcsec. Since the kinematic data range further inwards than the photometric data the SBprofile was extrapolated using a power-law; the fitted slope is -1.01 . This might overestimate the actual luminosity in the central regions since one might expect the $\mathrm{cD}$ galaxy NGC 315 to have a core with a shallower slope. Due to the outer cutoff of the SB-profile at $R=91.8$ arcsec, the total luminosity is an underestimate because the slope of the density profile at the cutoff of the data is still larger than -3 . This must then also be true for the $B_{\mathrm{T}}$ value from the RC3.

\section{2. $N G C 2434$}

The SB-profile is composite of $V$-band HST-data from Carollo et al. (1997) out to 10 arcsec and B-band groundbased data by Carollo \& Danziger (1994). The HST data were shifted by 0.88 mag to give a smooth transition between the two data sets. Note that Faber et al. (1989) give an effective radius of 81 arcsec whereas Rix et al. (1997) took 24 arcsec. In our analysis we used the latter value, but this is used only as a scaling constant and has no influence on any of the derived quantities.

\section{3. $N G C 3379$}

For this galaxy we used the CCD surface photometry of Davis et al. (1985) in both the $R$ - and $B$-bands.

\section{4. $N G C 4374$}

We used the photometry of Michard (1985). The innermost data point was removed.

\section{5. $N G C 5846$}

The $V$ band surface brightness profile of NGC 5846 was derived from observations performed in March 1999 at the ESO $2.2 \mathrm{~m}$ telescope equipped with the Wide Field Imager. This instrument covers a $34 \times 33 \mathrm{arcmin}^{2}$ field of 


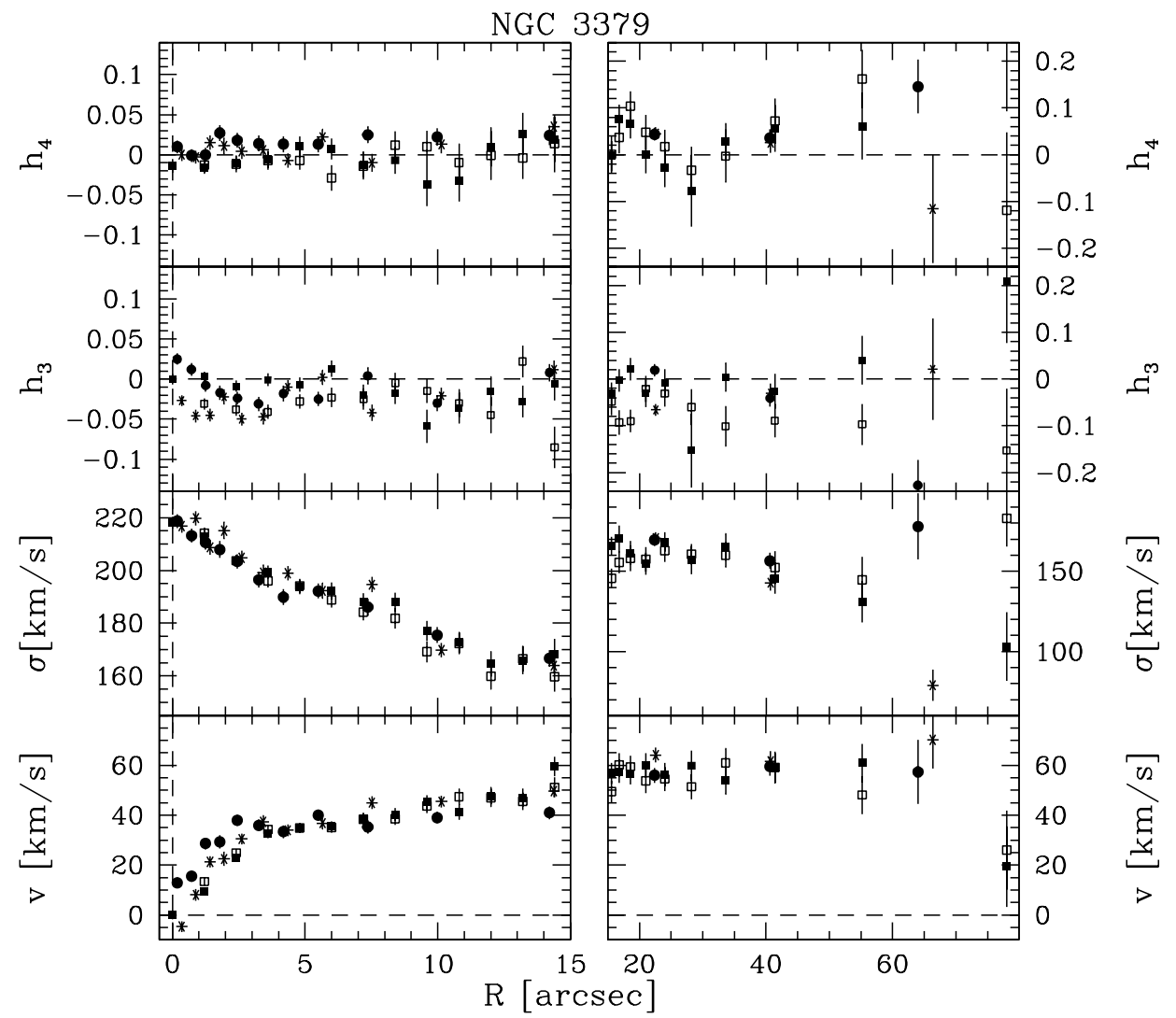

Fig. 4. The kinematics of NGC 3379 compared to the results of Statler \& Smecker-Hane (1999). From bottom to top: the folded mean velocity, velocity dispersion, $h_{3}$ and $h_{4}$ profiles. Stars and filled circles refer to the two sides of the galaxy and the major axis spectrum obtained here. Open and filled squares refer to the major axis data of Statler \& Smecker-Hane (1999). Note the changes of scales between the left and the right rows

view with a mosaic of $2 \times 4 \mathrm{CCDs}$ of $2 \mathrm{~K} \times 4 \mathrm{~K} 15 \mu \mathrm{m}$ pixels, with a scale of 0.238 arcsec/pixel. Six 5 minutes exposures were taken, shifted by 20 arcsecs in RA and Dec to fill the gaps between the CCDs. The reduction of the frames was performed under IRAF, using the mscred package to handle mosaic data. After the bias subtraction, sky flat-fielding, and frame combination (performed after the astrometric calibration) the resulting image is flat at the $1 \%$ level.

The isophote shape analysis was performed following Bender \& Möllenhoff (1987). The procedure was improved in two aspects. Mask files were produced automatically from the "segmentation frames" produced by the Sextractor program (Bertin \& Arnouts 1996). The surface brightness and integrated magnitude profiles following elliptical isophotes were computed from the averages of the unmasked pixels between the isophotes. Statistical errors were also derived from the measured rms. Radial profiles of surface brightness, ellipticity, position angle and higher order terms of the Fourier analysis are shown in Fig. 9. Note that the centers of the outer isophotes (at more than 500 arcsec from the center) are some 30 arcsec off the center of the inner ones. The surface brightness has been calibrated using the $V$-band photoelectric photometry of Colless et al. (1993). The comparison with data of Bender (unpublished) obtained with a small field of view CCD show good agreement up to 40 arcsec from the center, where the sky subtraction of Bender's data becomes unreliable.

\section{6. $N G C 7145$}

The SB-profile of Jørgensen et al. (1995) was used; the last two data points seem to suffer from sky subtraction errors and were dropped.

\section{7. $N G C 7192$}

For NGC 7192 HST photometry from Carollo et al. (1997) is available. These $V$-band data were shifted by $-0.47 \mathrm{mag}$ to merge smoothly into the ground-based $R$-band data from Carollo \& Danziger (1994). The HST-data were used out to 10.9 arcsec. 


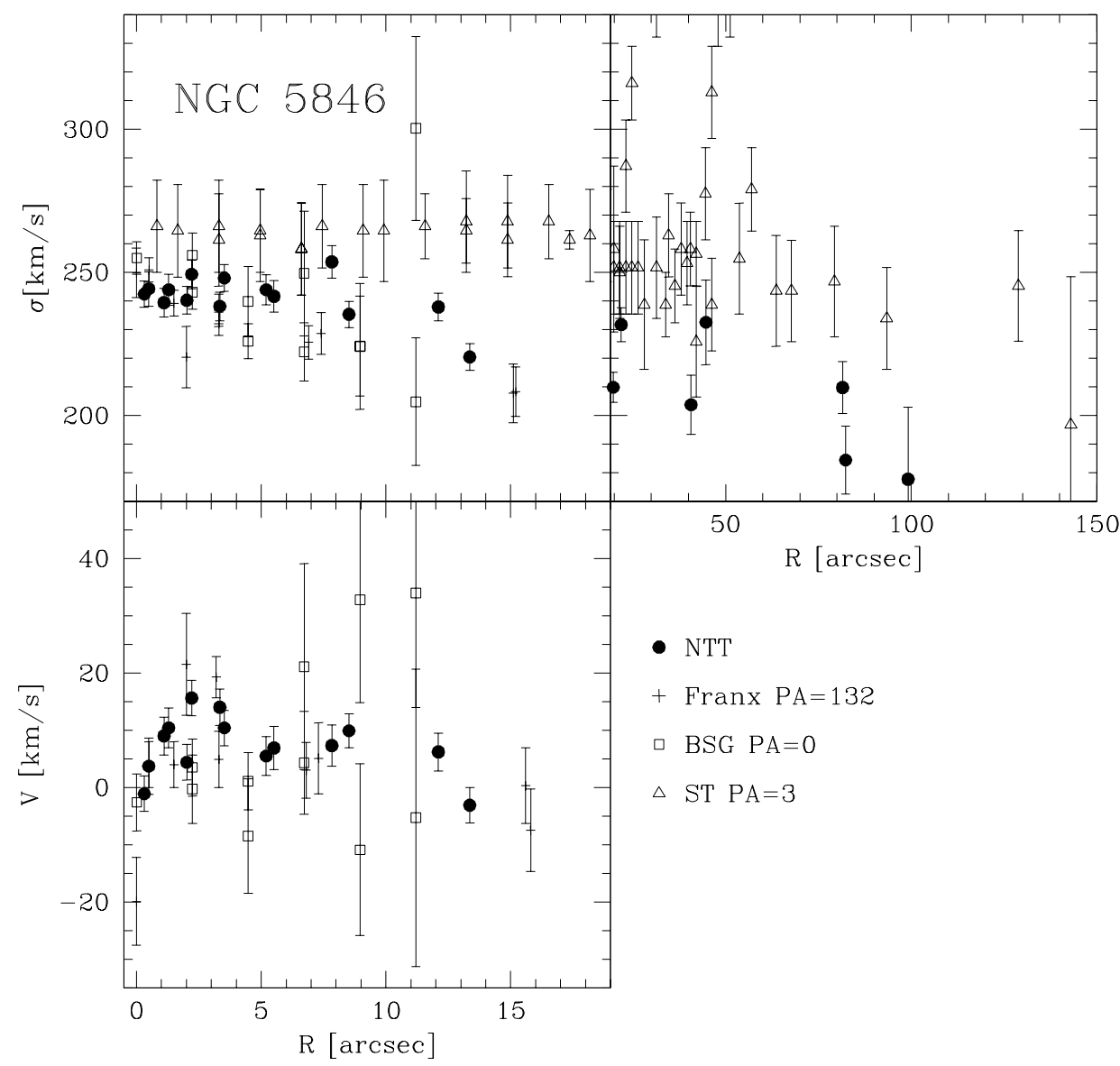

Fig. 5. Comparison with the velocity dispersion (top) and rotation velocities (bottom) of Franx et al. (1989, crosses), BSG94 (open squares), Sembach \& Tonry (1996, open triangles). The data of Carollo et al. (1993) have large error bars, within which they agree with our profiles. Note the change of scale at $19 \operatorname{arcsec}$

\section{8. $N G C 7507$}

The SB-profile is a composite of data from Bender (unpublished) and from Lauberts \& Valentijn (1989). The outermost data point was not used since it might be affected by sky-subtraction errors. Because the kinematic data reach further in towards the center than the photometry, we extrapolated using a power-law. The fitted central slope of the SB-profile is -0.15 .

\section{9. $N G C 7626$}

$B$-band ground-based data from King (1978) and Peletier et al. (1990) were merged with $V$-band HST-data (Bender, unpublished), after shifting the HST-data by $-0.6 \mathrm{mag}$.

\subsection{0. $N G C 3193$}

The SB-profile was taken from the electronically available tables of Goudfrooij et al. (1994). The profile was slightly oversmoothed to avoid high frequency fluctuations in the density profile.

\subsection{1. $N G C 3640$}

For this E2 galaxy the photometric data are from Goudfrooij et al. (1994). The data were extrapolated towards the center using a power-law to match the extent of the kinematic data; the fitted slope is -0.46 .

\subsection{2. $N G C 4168$}

We used photometric data of Caon et al. (1990) for this E1 galaxy. The profile was slightly oversmoothed to ensure a monotonically decreasing density profile.

\subsection{3. $N G C 4278$}

For this galaxy we used the SB-profile of Goudfrooij et al. (1994); this was oversmoothed compared to the GCV estimation (see Sect. 4) and extrapolated towards the center; the fitted central slope is -0.50 . 

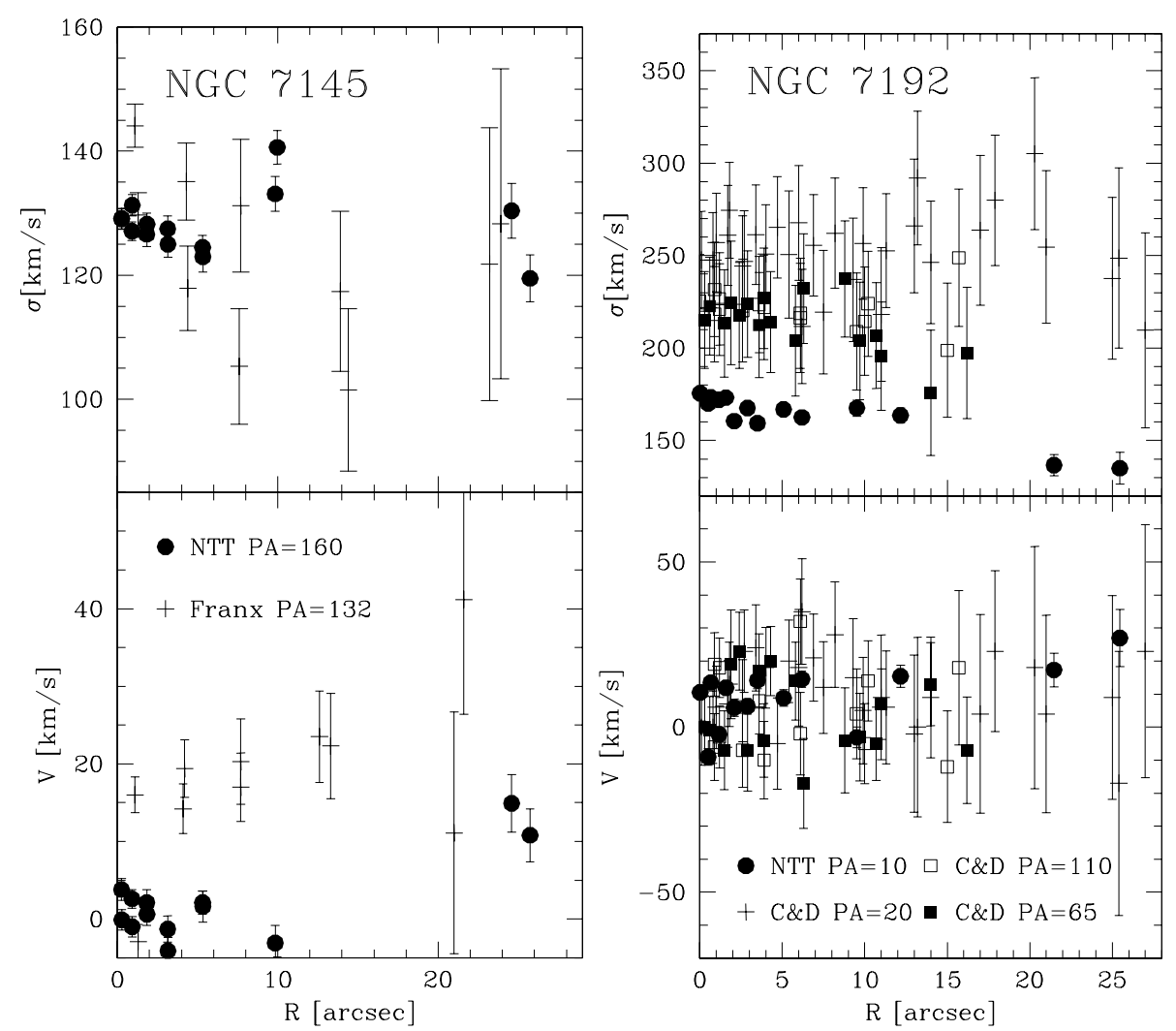

Fig. 6. Left: comparison with the velocity dispersion (top) and rotation velocities (bottom) of Franx et al. (1989, crosses) for NGC 7145. Right: comparison with the velocity dispersion (top) and rotation velocities (bottom) of Carollo \& Danziger (1994, major axis: crosses, minor axis: open squares, diagonal axis: open squares) for NGC 7192

\subsection{4. $N G C 4472$}

The total $B$-band magnitude of NGC 4472 and the effective radius $R_{\mathrm{e}}=104$ arcsec are comparable to the corresponding values for NGC 4486. The SB-data are taken from Caon et al. (1994).

\subsection{5. $N G C 4486$}

We used the photometry of Caon et al. (1990). For the effective radius we take the value of 110 arcsec given by Peletier et al. (1990).

\subsection{6. $N G C 4486 B$}

NGC $4486 \mathrm{~B}$ is a close companion of M 87 and is in some respects comparable to $\mathrm{M} 32$, the compact companion of M 31. There is an HST SB-profile available from Lauer et al. (1995) to which ground-based data from Prugniel (1997) were added after shifting by 0.6 mag.

\subsection{NGC 4494}

We used B-band photometry by Goudfrooij et al. (1994) for this E1 galaxy. The profile was extrapolated towards the center employing a power-law; the fitted slope is -0.57 .

\subsection{8. $N G C 4589$}

We used photometry by from Goudfrooij et al. (1994) and extrapolated the profile towards the center; the fitted slope is -0.50 .

\subsection{9. $N G C 4636$}

HST-photometry by Lauer et al. (1995) and ground-based data by Caon et al. (1994) were merged. The HST- $V$-band data were shifted by 1 mag to provide a smooth transition.

\section{Dynamical modelling}

\subsection{Modelling procedure}

The modelling technique follows the lines described in Gerhard et al. (1998; G+98) and Saglia et al. (2000; 


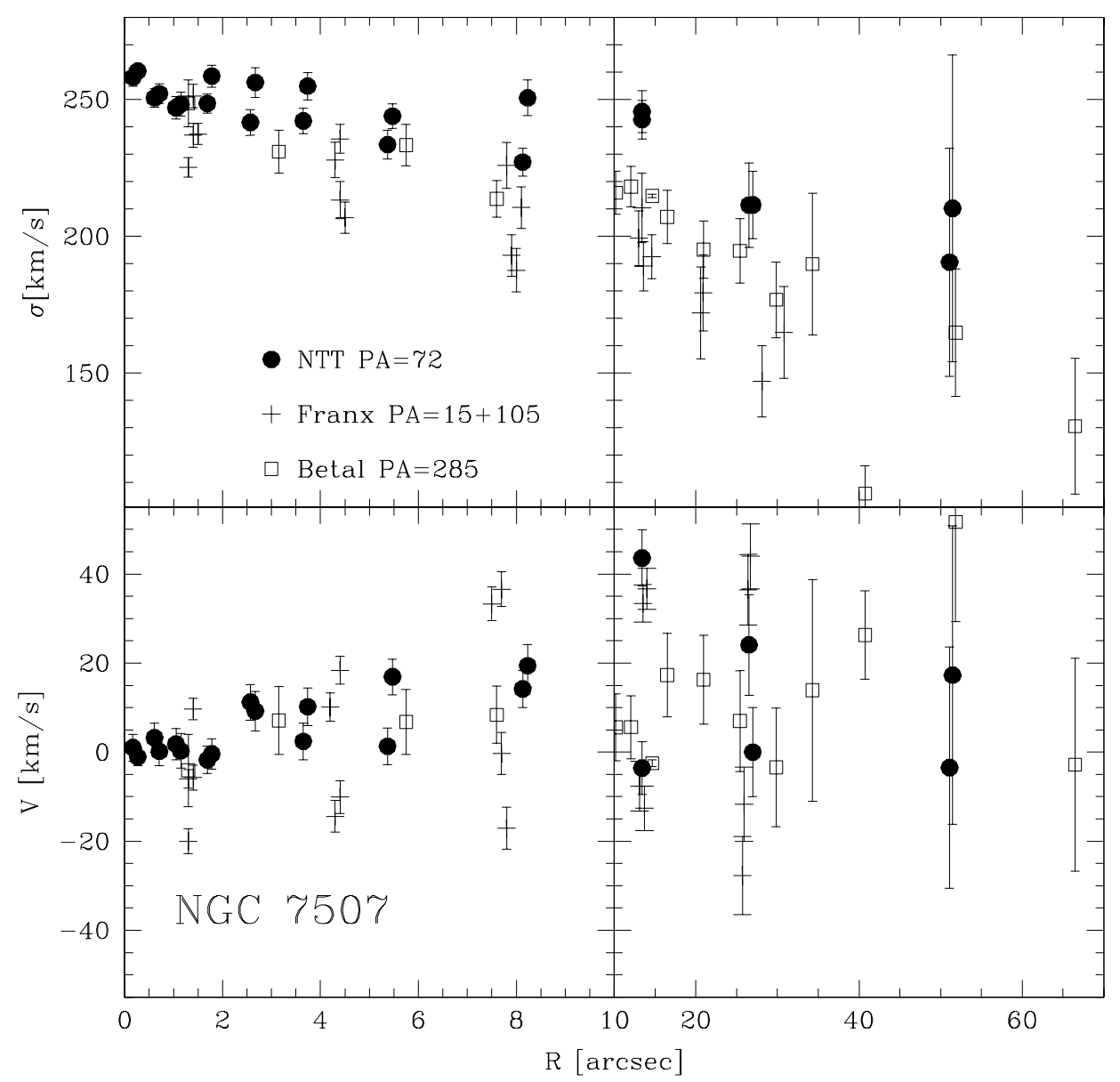

Fig. 7. Comparison with the velocity dispersion (top) and rotation velocities (bottom) of Franx et al. (1989, crosses), Bertin et al. (1994, open squares). Note the change of scale at 10 arcsec

S+2000), and consists of the following steps:

(i) Calculate the density and potential of the luminous matter from the smoothed SB-profile, assuming spherical symmetry and using a nonparametric regression algorithm based on generalized cross validation (GCV, Wahba \& Wendelberger 1980).

(ii) Specify a gravitational potential, consisting of the potential of the luminous matter and a nonsingular isothermal dark matter halo of the form

$\Phi^{\mathrm{DM}}=\frac{1}{2} v_{0}^{2} \ln \left(r^{2}+r_{0}^{2}\right)$

with core radius $r_{0}$ and circular speed $v_{0}$. By employing these halo potentials we implicitly maximize the luminous mass in the galaxy.

(iii) In a given potential, construct a set of basis functions for the phase-space distribution (DF), each reproducing the stellar density. The basis functions contain the isotropic model, a radially anisotropic model, and several series of tangentially anisotropic functions. The form of these basis functions is described in $\mathrm{G}+98$.

(iv) Project each of these basis DFs into the space of kinematic observables. (v) Determine the smoothing parameter $\lambda$ and the cumulative $\chi^{2}$ distribution from Monte Carlo simulations of kinematic data similar to those for the galaxy under study.

(vi) Fit the composite sum of the basis DFs in the kinematic observables to the measured velocity dispersions $\sigma$ and Gauss-Hermite parameters $h_{4}$ of the galaxy, by a regularised least-square algorithm imposing non-negativity and smoothness of the composite DF. In this way we find the unique best DF for the given potential and the previously fixed $\lambda$.

(vii) By varying the parameters of the dark matter component and repeating steps (ii-vi) determine the range of potentials consistent with the data, using a $\chi^{2}$-statistic.

In this procedure, it is advantageous to use a realistic model DF for setting the velocity scales with which the projected Gauss-Hermite moments of the basis DFs are computed. For this we have either taken the isotropic model or a radially anisotropic DF determined by a parametric fit. In most cases we have used "basis 2", which consists of 59 functions including this radially anisotropic model. "Basis 1" consists of the same 59 functions, but in this case the isotropic model was used for the velocity scales. Finally "basis 3" does not contain 


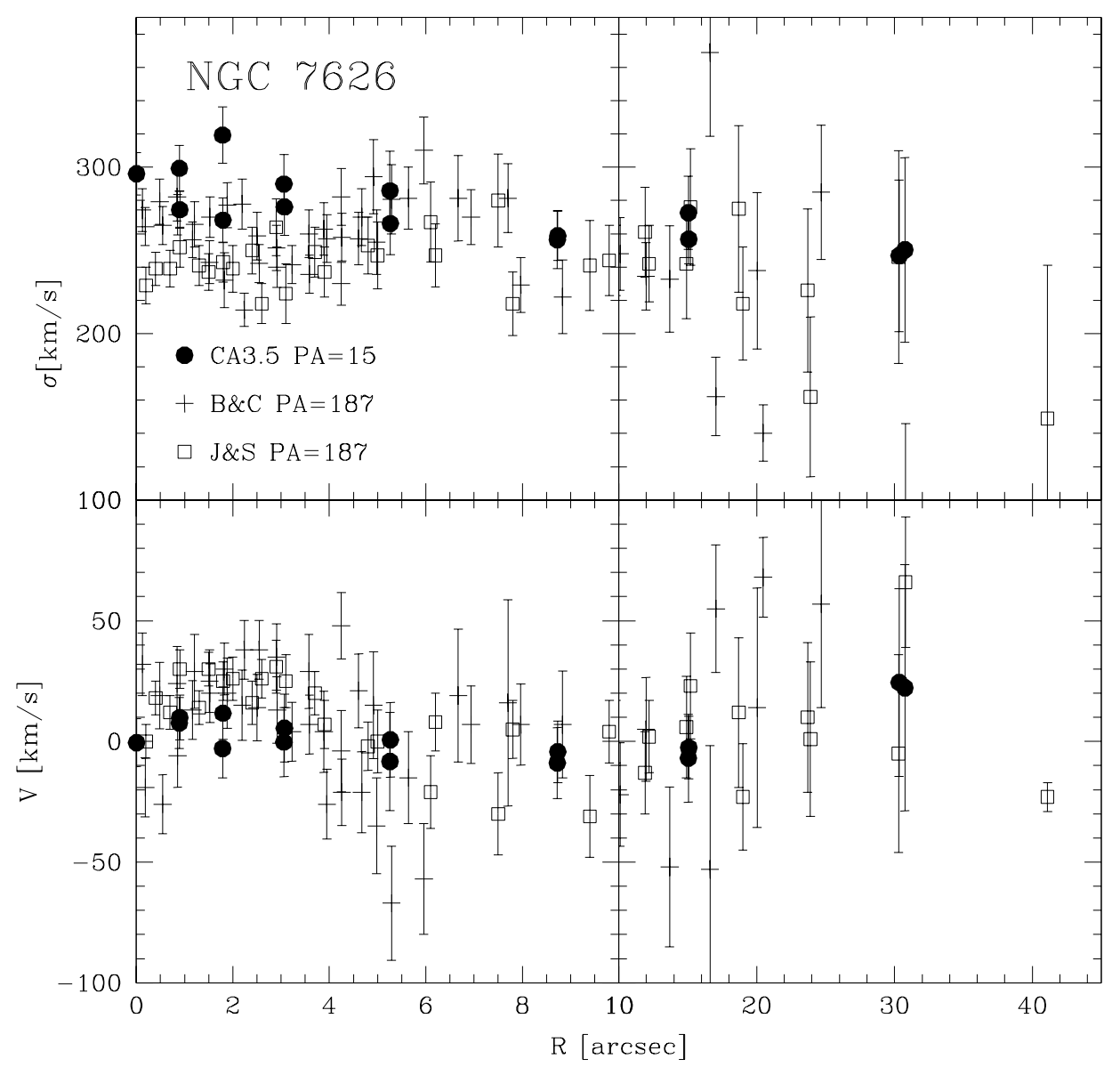

Fig. 8. Comparison with the velocity dispersion (top) and rotation velocities (bottom) of Balcells \& Carter (1993, crosses), Jedrzejewski \& Schechter (1989, open squares). Note the change of scale at 10 arcsec

the radially anisotropic function and thus has only 58 components. Which basis was used for which galaxy is shown in Tables 5 and 6. NGC 1399 was modelled using a similar but not identical basis as described in $\mathrm{S}+2000$. For this galaxy we have also verified the robustness of the kinematic results by repeating the analysis with a very different set of basis functions, consisting of the isotropic and 39 radially anisotropic models; see Sect. 5.3.

In the non-parametric fit the degree of smoothness is directed by a penalty term whose weight $\lambda$ is determined by Monte Carlo simulations. For each of a series of values for $\lambda$, we analyse 100 Monte Carlo data sets. These datasets are generated from a parametric model which matches the galaxy data closely, and kinematic data points are generated as Gaussian random deviates with the observed error bars at the positions of the observed data points. The value of $\lambda$ which results in the smallest rms relative deviation between the known DF of the input model and the recovered DF, averaged over the 100 realizations, was chosen for the final fits (see $\mathrm{G}+98$ and $\mathrm{S}+2000$ ). Here the rms deviation between input and output DFs was determined on a grid out to three times the last kinematic data point. It turned out that $\lambda$ is only weakly dependent on the potential. The final values of $\lambda$ employed for our galaxies are given in Tables 5 and 6 .

\subsection{Estimation of confidence intervals}

We estimate the confidence interval from the Monte Carlo simulations described above, for the final value of $\lambda$ (see also $\mathrm{G}+98$ ). The $95 \%$ confidence level is taken to be at the value of $\chi_{\sigma+h_{4}}^{2}$ which is surpassed only by 5 of the 100 realizations. The results from this procedure do not depend much on whether the selfconsistent potential or one with a halo is used for the Monte Carlo simulations, and the average value from these two cases is used.

As in $\mathrm{S}+2000$ we have encountered the problem of high values of $\chi^{2}$ (>1 per data point) for some of the galaxies, in particular those with the new, higher quality data. The reason is the same as described in that paper: the point to point variations in the real data are larger than the error bars derived from the simulations of the spectra. These point-to-point variations in the data appear to be caused by systematic effects in the data.

To obtain smaller values of the $\chi^{2}$-statistic one can decrease the degree of smoothing on the DF. We have tested 
Table 3. Photometry and distances for all galaxies in the sample. Column 1 gives the NGC number, Col. 2 the morphological type, Col. 3 the adopted distance, Col. 4 the total integrated magnitude as given in the RC3 (de Vaucouleurs et al. 1991), Col. 5 the effective radius in arcsec, Col. 6 the source of the effective radius (b Bicknell 1989, bs BSG94, f Faber et al. 1989, g Gerhard et al. 1998, p Peletier et al. 1990, r Rix et al. 1997), Col. 7 the photometric band, Col. 8 the source of the photometric data: Be is Bender (unpublished), B Bicknell et al. (1989), C90 Caon et al. (1990), C94 Caon et al. (1994), C Carollo et al. (1994, 1997), Da Davis et al. (1985), D Djorgovski (1985), GS Gerhard \& Saglia (Sect. 3.5), G98 Gerhard et al. (1998), G Goudfrooij et al. (1994), J Jørgensen et al. (1995), Ke Kent (1984), K King (1978), L Lauer et al. (1995), LV Lauberts \& Valentijn (1989), M Michard (1985), P Peletier et al. (1990), P97 Prugniel (1997). Where two author abbreviations are given separated by a comma, the first is for ground based and the second for HST data. Author abbreviations separated by a "+" signify that their data were merged. Columns 9 and 10 are the inner and outer boundaries of the photometry in units of the effective radius, Cols. 11 and 12 similarly for the kinematic data. In case of NGC 3379 S denotes the data from Statler \& Smecker-Hane (1999)

\begin{tabular}{|c|c|c|c|c|c|c|c|c|c|c|c|}
\hline NGC & type & $d[\mathrm{Mpc}]$ & $B_{\mathrm{T}}$ & $R_{\mathrm{e}}\left[^{\prime \prime}\right]$ & Source & Band & Source & $R_{\min }^{\mathrm{phot}}\left[R_{\mathrm{e}}\right]$ & $R_{\max }^{\mathrm{phot}}\left[R_{\mathrm{e}}\right]$ & $R_{\min }^{\mathrm{kin}}\left[R_{\mathrm{e}}\right]$ & $R_{\max }^{\mathrm{kin}}\left[R_{\mathrm{e}}\right]$ \\
\hline 315 & $\mathrm{E} 2, \mathrm{cD}$ & 77.6 & -22.25 & 68 & $\mathrm{p}$ & $B$ & $\mathrm{P}$ & 0.056 & 1.4 & 0.002 & 1.13 \\
\hline 1399 & $\mathrm{E} 1, \mathrm{cD}$ & 21.9 & -21.15 & 42 & $\mathrm{~b}$ & $B$ & $\mathrm{~B}, \mathrm{~L}$ & 0.0010 & 75.3 & 0.002 & 2.31 \\
\hline 2434 & E0-1 & 30.3 & -20.08 & 24 & $\mathrm{r}$ & $B, V$ & $\mathrm{C}, \mathrm{C}$ & 0.0006 & 6.3 & 0.006 & 2.58 \\
\hline 3379 & E1 & 13.2 & -20.36 & 35 & $\mathrm{f}$ & $B, R$ & Da & 0.04 & 4.5 & 0.005 & 2.95 \\
\hline $\mathrm{S}$ & & & & & & & & & & 0.0 & 2.23 \\
\hline 4374 & E1 & 20.5 & -21.47 & 57 & $\mathrm{p}$ & $B$ & M & 0.009 & 7.8 & 0.005 & 1.17 \\
\hline 5846 & E0 & 35.9 & -21.73 & 83 & $\mathrm{f}$ & $V$ & GS & 0.0 & 6.7 & 0.004 & 1.19 \\
\hline 6703 & E0 & 28.0 & -19.92 & 30 & $\mathrm{~g}$ & $B$ & G98 & 0.003 & 2.4 & 0.006 & 2.61 \\
\hline 7145 & E0 & 28.4 & -20.23 & 39 & $\mathrm{f}$ & $B$ & $\mathrm{~J}$ & 0.003 & 2.8 & 0.007 & 1.66 \\
\hline 7192 & E0 & 37.0 & -20.65 & 34 & $\mathrm{f}$ & $R$ & $\mathrm{C}, \mathrm{C}$ & 0.0004 & 5.9 & 0.0006 & 0.75 \\
\hline 7507 & E0 & 26.9 & -20.79 & 31 & $\mathrm{f}$ & $B$ & $\mathrm{Be}+\mathrm{LV}$ & 0.009 & 4.1 & 0.005 & 1.66 \\
\hline 7626 & E1 pec & 55.1 & -21.55 & 38 & $\mathrm{f}$ & $B$ & $\mathrm{~K}+\mathrm{P}, \mathrm{Be}$ & 0.0003 & 1.8 & 0.003 & 2.10 \\
\hline 3193 & E2 & 37.8 & -21.06 & 24 & bs & $B$ & $\mathrm{G}$ & 0.04 & 2.7 & 0.03 & 1.41 \\
\hline 3640 & $\mathrm{E} 2$ & 35.1 & -21.37 & 33 & bs & $B$ & G & 0.03 & 2.8 & 0.02 & 0.63 \\
\hline 4168 & E1 & 41.8 & -21.00 & 45 & bs & $B$ & C90 & 0.005 & 5.4 & 0.01 & 0.67 \\
\hline 4278 & E1 & 22.6 & -20.68 & 33 & bs & $B$ & G & 0.03 & 2.9 & 0.02 & 0.45 \\
\hline 4472 & E1 & 20.5 & -22.19 & 104 & bs & $B$ & C94 & 0.006 & 12.5 & 0.005 & 0.54 \\
\hline 4486 & E0 & 20.5 & -21.97 & 110 & $\mathrm{p}$ & $B$ & $\mathrm{C} 90$ & 0.003 & 8.9 & 0.02 & 0.39 \\
\hline $4486 \mathrm{~B}$ & $\mathrm{cE} 1$ & 20.5 & -17.20 & 3.1 & bs & $V$ & P97,L & 0.007 & 6.5 & 0.2 & 2.79 \\
\hline 4494 & E1 & 10.7 & -19.44 & 45 & bs & $B$ & G & 0.02 & 1.8 & 0.01 & 0.71 \\
\hline 4589 & $\mathrm{E} 2$ & 46.6 & -21.65 & 41 & bs & $B$ & G & 0.03 & 2.2 & 0.02 & 0.77 \\
\hline 4636 & E0 & 20.5 & -21.13 & 102 & bs & $B$ & $\mathrm{C} 94, \mathrm{~L}$ & 0.0001 & 6.4 & 0.02 & 0.33 \\
\hline
\end{tabular}

this for NGC 7626. In this case, the range of circular velocities at the last kinematic data point, $v_{\mathrm{c}}\left(R_{\max }\right)$, determined from the fitting procedure with the best value of the smoothing parameter $\lambda$ as above, is $\sim 370-440 \mathrm{~km} \mathrm{~s}^{-1}$ at $95 \%$ confidence (see below). Without any smoothing $(\lambda=0)$, these models can be fitted with $\chi^{2} \simeq 0.7$ per point, and models with $v_{\mathrm{c}}\left(R_{\max }\right)$ down to $350 \mathrm{~km} \mathrm{~s}^{-1}$ and up to more than $500 \mathrm{~km} \mathrm{~s}^{-1}$ are consistent with the data $\left(\chi^{2}<1.27\right)$. If $\lambda$ is chosen such that the best-fitting models have $\chi^{2} \simeq 1.0$, then a range in $v_{\mathrm{c}}\left(R_{\max }\right)$ from $\simeq 360 \mathrm{~km} \mathrm{~s}^{-1}$ to $\sim 500 \mathrm{~km} \mathrm{~s}^{-1}$ have $\chi^{2}<1.27$, i.e., are consistent with the data at the $2 \sigma$ level. However, the price to be paid is a much less smooth DF. In the last case, the DF has several holes in radius (energy): in one example, it tends to zero at some small radius and at some large radius, and in addition dives by more than an order of magnitude relative to nearby regions at a number of other energies. For $\lambda=0$, the behaviour of the DF is even worse; in this case it is highly unsmooth, as expected, and has of order 5 holes in energy where it tends to zero. Such
DFs are contrary to the smooth coarse-grained DFs of hot stellar systems that are predicted by violent relaxation. Moreover, we believe that decreasing $\lambda$ in order to improve the point-by-point fit of the dynamical models is also inappropriate because the point-to-point variations in the data often appear to be caused by systematic effects, such as line emission, dust absorption, residual template mismatches, and asymmetries between the two sides of the galaxy.

To derive a confidence interval despite the presence of systematic errors in the data, we have in most cases (see also $\mathrm{S}+2000$ ) (i) increased the kinematic errors bars by a global factor until one obtains $\chi^{2} \simeq 1$ per data point for the best model, (ii) used this revised data set for the Monte Carlo simulations as described above, giving a new value for the $95 \%$ value of $\chi^{2}$, and (iii) finally rescaled this value upwards by the factor of the error enlargement in order to compare with the fits based on the original errors. These (where necessary) rescaled confidence values and the resulting confidence intervals are shown in Figs. 10 
Table 4. Calibration checks for the adopted SB-profiles. Column 1 is the NGC number; Col. 2 gives the total $B$-magnitude from the RC3 (de Vaucouleurs et al. 1991); Col. 3 the aperture of the photoelectric measurements of Burstein et al. (1987), Col. 4 the corresponding aperture magnitude corrected for extinction and converted to the adopted distance (Table 3). Column 5 gives the integral of the adopted SB-profiles within the aperture of Burstein et al. (1987), and Col. 6 the difference between the integrated and the measured aperture magnitudes. Two galaxies, NGC 3193 and NGC 4486B, are not in the catalogue of Burstein. Column 7 gives the integrated magnitude of the SB-profiles out to $1 R_{\mathrm{e}}-0.75$ mag. For NGC 1399 and NGC 7507 the SB-profiles were corrected to the Burstein et al. photometric system

\begin{tabular}{rrrrrrr}
\hline NGC & $B_{\mathrm{T}}^{\mathrm{RC} 3}$ & $R_{\mathrm{A}}\left[^{\prime \prime}\right]$ & $B\left(<R_{\mathrm{A}}\right)$ & $B_{\text {int }}$ & $B_{\text {int }}-B\left(<R_{\mathrm{A}}\right)$ & $B_{\text {int }}\left(R_{\mathrm{e}}\right)-0.75$ \\
\hline 315 & -22.25 & 30.00 & -21.42 & -21.45 & -0.03 & -22.80 \\
1399 & -21.15 & 45.41 & -20.40 & -20.69 & -0.29 & -21.11 \\
2434 & -20.08 & 47.55 & -20.32 & -20.30 & 0.02 & -20.62 \\
3193 & -21.06 & - & - & - & - & -20.96 \\
3379 & -20.36 & 47.55 & -19.54 & -19.67 & -0.13 & -20.20 \\
3640 & -21.37 & 42.38 & -20.85 & -20.72 & 0.13 & -21.32 \\
4168 & -21.00 & 78.91 & -20.63 & -20.73 & -0.10 & -21.17 \\
4278 & -20.68 & 47.55 & -20.09 & -20.09 & 0.00 & -20.63 \\
4374 & -21.47 & 42.38 & -20.38 & -20.37 & 0.01 & -21.32 \\
4472 & -22.19 & 78.91 & -21.25 & -21.38 & -0.13 & -22.35 \\
4486 & -21.97 & 47.55 & -20.67 & -20.70 & -0.03 & -22.07 \\
$4486 \mathrm{~B}$ & -17.20 & - & - & - & - & -17.41 \\
4494 & -19.44 & 78.91 & -18.94 & -18.98 & -0.04 & -19.39 \\
4589 & -21.65 & 64.14 & -21.28 & -21.23 & 0.05 & -21.70 \\
4636 & -21.13 & 78.91 & -20.44 & -20.53 & -0.09 & -21.51 \\
5846 & -21.73 & 33.66 & -20.52 & -20.51 & 0.01 & -21.88 \\
6703 & -19.92 & 42.38 & -19.77 & -19.79 & -0.02 & -20.35 \\
7145 & -20.23 & 47.55 & -19.62 & -19.59 & 0.03 & -20.24 \\
7192 & -20.65 & 47.55 & -20.15 & -20.15 & 0.00 & -20.73 \\
7507 & -20.79 & 33.66 & -20.29 & -19.91 & 0.38 & -21.01 \\
7626 & -21.55 & 78.91 & -21.26 & -21.37 & -0.11 & -21.70 \\
\hline
\end{tabular}

Table 5. Dynamical modelling parameters for the part of the sample galaxies with new kinematic data. NGC 1399 analyzed with the identical method but another basis set in S+2000 is included for completeness. Column 1 gives NGC number, Col. 2 the optimal value of $\lambda$, Col. 3 codes the employed basis, Col. 4 lists the resulting rms accuracy with which a smooth DF can be recovered from the data, Col. 5 gives the (probably systematic) error enlargement factor, Cols. 6 and 7 the alternative systematic error floors to achieve a $\chi^{2}=1$ per data point fit. Systematic errors in $\sigma$ are given in $\mathrm{km} \mathrm{s}^{-1}$. See Sect. 4 for details

\begin{tabular}{lrrrrrr}
\hline NGC & $\lambda$ & basis & rmsDF & err. & sys. $\sigma$ & sys. $h_{4}$ \\
\hline 315 & 0.01 & 1 & 0.125 & $43 \%$ & 6 & 0.027 \\
1399 & 0.02 & - & 0.126 & $40 \%$ & 6 & 0.01 \\
2434 & 0.02 & 2 & 0.134 & 0 & 0 & 0 \\
$3379 \mathrm{~B}$ & 0.02 & 2 & 0.116 & 0 & 0 & 0 \\
$3379 \mathrm{R}$ & 0.01 & 2 & 0.116 & 0 & 0 & 0 \\
4374 & 0.03 & 2 & 0.118 & $35 \%$ & 3 & 0.011 \\
5846 & 0.02 & 3 & 0.136 & $8 \%$ & 0 & 0 \\
6703 & 0.006 & 2 & 0.176 & $10 \%$ & 1 & 0.01 \\
7145 & 0.002 & 2 & 0.159 & $80 \%$ & 7 & 0.008 \\
7192 & 0.006 & 3 & 0.156 & 0 & 0 & 0 \\
7507 & 0.03 & 2 & 0.116 & $13 \%$ & 3 & 0.005 \\
7626 & 0.03 & 2 & 0.123 & $35 \%$ & 5.5 & 0.01 \\
\hline
\end{tabular}

Table 6. Same as Cols. 1-5 of Table 5, for the sample galaxies with kinematic data from BSG94. Larger values of the RMS deviation of the DF indicate poorer quality of the kinematic data (both in terms of sampling and in terms of the sizes of the error bars). Especially NGC 4486B is less reliable

\begin{tabular}{lrrrr}
\hline NGC & $\lambda$ & basis & rmsDF & err. \\
\hline 3193 & 0.01 & 2 & 0.181 & 0 \\
3640 & 0.01 & 2 & 0.129 & $30 \%$ \\
4168 & 0.01 & 2 & 0.168 & 0 \\
4278 & 0.03 & 3 & 0.097 & $50 \%$ \\
4472 & 0.03 & 2 & 0.084 & $10 \%$ \\
4486 & 0.01 & 1 & 0.115 & 0 \\
$4486 \mathrm{~B}$ & 0.03 & 2 & 0.281 & 0 \\
4494 & 0.02 & 2 & 0.107 & $60 \%$ \\
4589 & 0.002 & 2 & 0.170 & 0 \\
4636 & 0.1 & 2 & 0.112 & 0 \\
\hline
\end{tabular}




\section{NGC 5846}
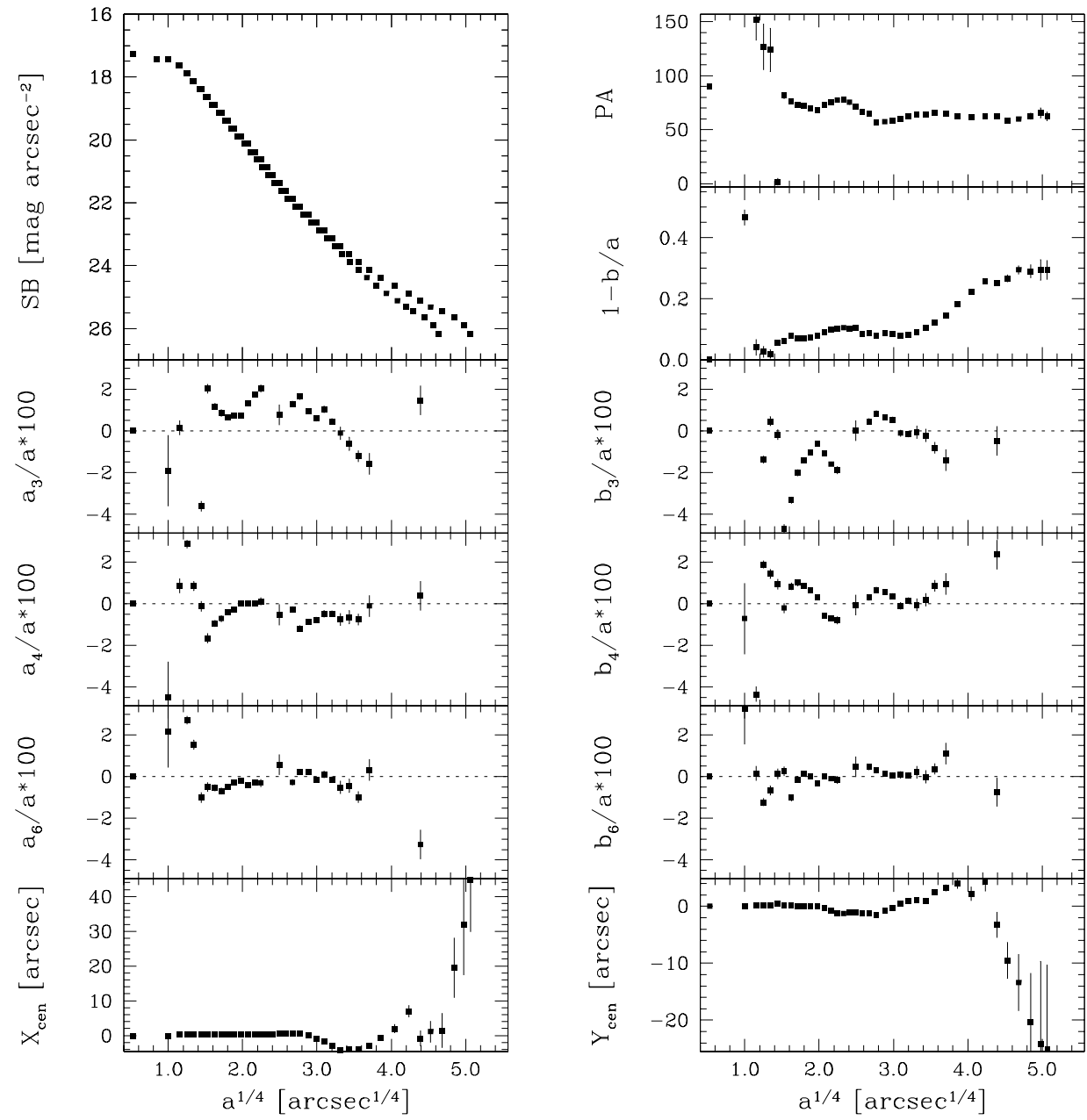

Fig. 9. The $V$-band surface brightness and isophote shape parameters of NGC 5846 as a function of the $1 / 4$ power of the semimajor distance from the center

and 11. Tables 5 and 6 list the necessary error enlargements.

We have also investigated an alternative way of treating the problem, based on the observation that, in terms of the respective error bars, large point-to-point variations between neighbouring data points occur most often in those galaxies with new data and small errors, and within these in the central parts where the error bars are particularly small. This suggests that systematic effects influence all data points in nearly the same way and with a similar amplitude. To model this, we have for these galaxies, both in the model fits and in the Monte Carlo simulations, added in quadrature a constant error in $\sigma$ and $h_{4}$ to the statistical errorbars, until the best-fitting models reached $\chi^{2}=1$ per point. The resulting "systematic" errors are rather small, ranging from 3 to $7 \mathrm{~km} \mathrm{~s}^{-1}$ in $\sigma$ and 0.005 to 0.027 in $h_{4}$. Table 5 gives values for individual galaxies. For some objects, we have done the full analysis with both methods, and generally find rather little difference between the results. See Sect. 5.3 for a comparison plot.
Below, we present our results using the first technique, except in the two galaxies NGC 315 and NGC 7626 where the second method gave somewhat better results.

\section{Results}

For each of the sample galaxies we calculated dynamical models using the method described above, for a series of potentials (between 20 and 150 per galaxy). A given potential can be parametrized by the value of the circular velocity at the radius of the last kinematic data point, $v_{\mathrm{c}}\left(R_{\max }\right) \cdot \chi^{2}$ diagrams (plots of resulting model $\chi^{2}$ against $\left.v_{\mathrm{c}}\left(R_{\max }\right)\right)$ together with confidence intervals as described in Sect. 4.2 are shown in Figs. 10 (for the sample with new kinematic data) and 11 (for the sample with spectroscopic data from BSG94). This grouping is maintained in subsequent plots because of the different radial range covered by the two sets of kinematic data. From these figures we select three models for each galaxy: two from the boundaries 


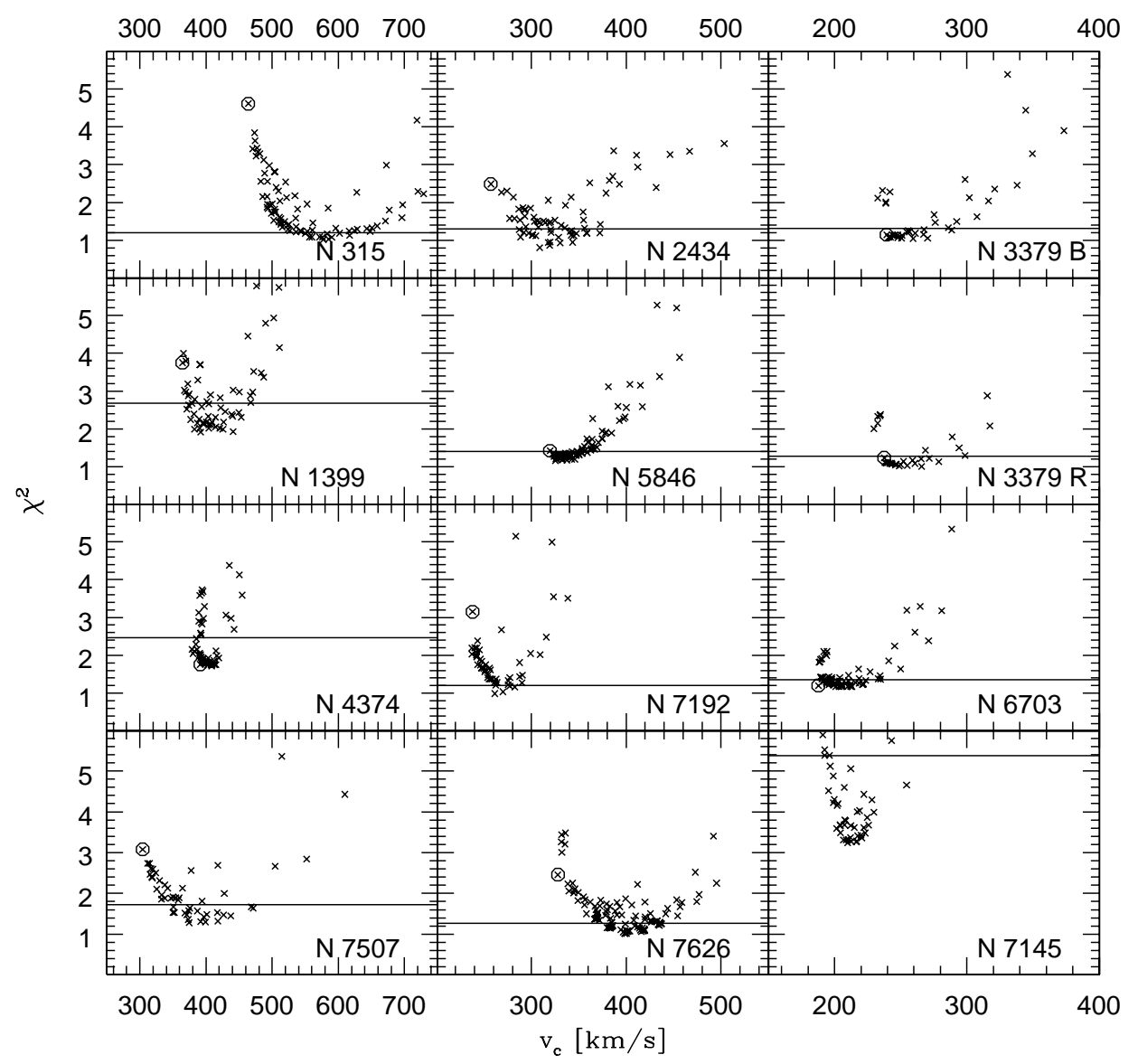

Fig. 10. $\chi^{2}$ versus circular velocity. Horizontal lines indicate the (rescaled) $95 \%$ confidence levels. Models lying above that line are inconsistent with the kinematic data. The SC models are encircled. For NGC 7145 it lies outside the plot. Note that the scale of the $v_{\text {circ }}$ axes is different for each column

of the confidence interval and one of the best-fitting models from the middle of the confidence range. These models are depicted with the same linestyles in all the plots to follow: dashed lines for the two boundary models, and a solid line for the respective "best" model. Note that this "best" model does not necessarily have the smallest value of $\chi^{2}$ since it is chosen in the middle of the range of valid models. In figures showing model fits to the kinematic data, or in circular velocity curve plots, the self-consistent (SC) models are additionally shown as dotted lines.

Figures 12 to 17 present the model fits to the kinematic data for all galaxies analysed. For each galaxy three panels are shown: the upper panel shows the velocity dispersion $\sigma$, the middle panel shows the $h_{4}$ parameter, and the lower panel shows the intrinsic anisotropy parameter $\beta$ defined as

$$
\beta=1-\frac{\sigma_{\mathrm{t}}^{2}}{\sigma_{\mathrm{r}}^{2}}
$$

where $\sigma_{\mathrm{t}}$ and $\sigma_{\mathrm{r}}$ are the one-dimensional tangential and radial velocity dispersion components.

The derived anisotropy profiles are surprisingly uniform. For most galaxies, the best-fit models are moderately anisotropic at $R_{\mathrm{e}} / 2$, with typical $\beta \lesssim 0.3$, in a few cases $\beta \lesssim 0.5$. Towards the center most turn towards isotropy; a clear exception is NGC 2434. In a number of galaxies the transition to a near-isotropic center occurs in the inner $3^{\prime \prime}$ and is uncertain because of the low spatial resolution of the data; in others, however, it starts at $R \simeq 10^{\prime \prime}$ and is therefore real (NGC 4374, 3379, 4168, 4472, 4486). We have also checked, using the data for NGC 1399, that the shape of the inner anisotropy profile is not dependent on the set of basis functions used in the modelling; see Fig. 12 and Sect. 5.3. In the outer parts, the $\beta$-profiles also generally turn back towards isotropy or even tangential anisotropy, but due to the finite range of the kinematic data, the detailed values near the outer edge depend on the halo mass distribution and the errors on $\beta$ there are large.

The circular velocity curves (CVCs) resulting from these model potentials are shown in Figs. 18 and 19. They are plotted as a function of $R / R_{\mathrm{e}}$, and extend to the radius of the outermost kinematic data point. All CVCs are consistent with being flat outside $R / R_{\mathrm{e}} \sim 0.3$ at the $\sim 10 \%$ level. Extended flat rotation curves, however, can only be demonstrated for about eight of the galaxies with new kinematic data. In some of these, e.g., NGC 1399, 3379, 


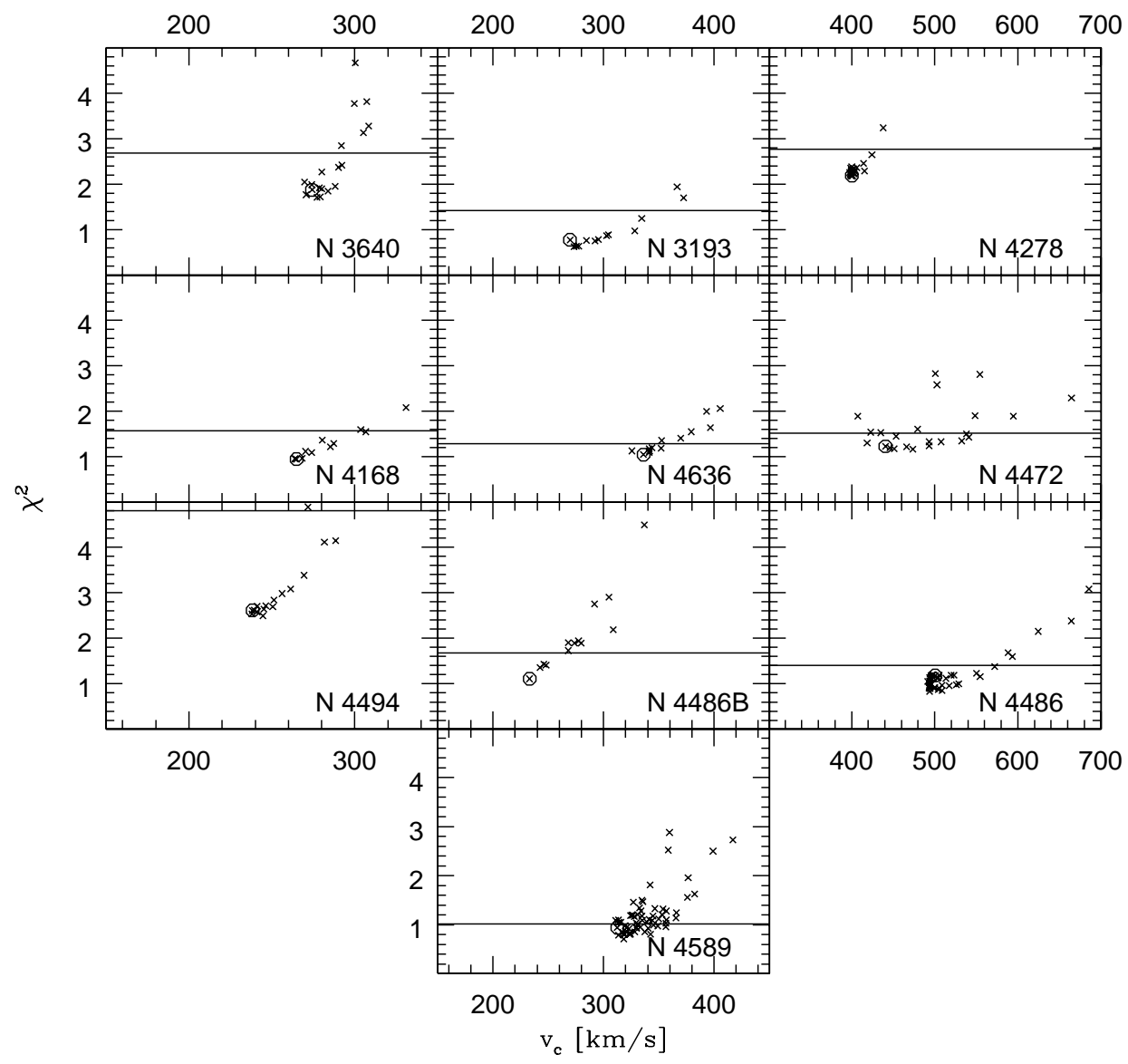

Fig. 11. $\chi^{2}$ versus circular velocity for the galaxies with kinematics from BSG94. Horizontal lines indicate the (rescaled) $95 \%$ confidence levels. Models lying above that line are inconsistent with the kinematic data. The SC models are encircled

the CVC rises to a maximum at $R / R_{\mathrm{e}} \sim 0.3 \sim 10^{\prime \prime}$, before falling by about $10 \%$ to reach the flat part at $R / R_{\mathrm{e}} \sim 1$. In others, e.g., NGC 2434, 7626, no such maximum is seen before the flat part of the $\mathrm{CVC}$ is reached. In some, e.g., NGC 315, the situation is unclear because the data do not extend far enough. The same is true for many of the galaxies from the BSG94 sample, where also in some cases the poorer quality of the data (e.g., in NGC 4486B) causes additional uncertainty.

Figures 20 and 21 show the corresponding cumulative mass-to-light ratios in the $B$-band for all sample galaxies. For each case, the permitted range is indicated by plotting the two boundary models and the "best" model from the central region of the confidence interval. Numerical values for the central and total mass-to-light ratios within the range of the kinematic data are given in Table 7 .

For all galaxies, the respective "best" model shows an outward increase of $M / L$. However, the increase ranges from very small (NGC 3379) to about a factor of 2 (NGC 2434). Because of the finite radial range of the data, the modelling will allow some artificially massive halo models (see G+98 for an explanation). Three of the galaxies in Fig. 20 (NGC 3379, NGC 4374, NGC 6703) are consistent with a flat $M / L$ over the range of even the extended kinematic data (the self-consistent constant$M / L$ model lies inside the $95 \%$-confidence line), although in each case the $M / L$ of the "best" model increases outwards. Both in NGC 1399, NGC 5846 the constant$M / L$ model lies outside the $95 \%$-confidence line. In NGC 5846 we consider this only marginal evidence, given the small radial range covered by the data and the similarity between the models. In NGC 1399 the weak evidence for dark matter is confirmed by X-ray and other data; see below. The rising $M / L$ shown by the models in NGC 7145 is influenced strongly by this galaxy's peculiar inner kinematics. The clearest candidates for radially increasing mass-to-light ratios in this sample are therefore NGC 315, NGC 2434, NGC 7507, and NGC 7626, where in each case the self-consistent model is a poor fit to the data.

In Table 8 we compare our derived mass-to-light ratios with those determined by van der Marel (1991), Lauer (1985) and Saglia et al. (1992). The values given by the first author were transformed to the $B$-band, assuming a mean extinction-corrected Johnson colour of $B-R=1.80$. All values were scaled to the distances 
Table 7. Circular velocities at the outermost kinematic data point, central $B$-band $M / L$ ratios, and ratios of the $B$-band $M / L$ at the outermost kinematic data point over the central value. All data are given for the "best-fitting" model as well as for two models from both sides of the confidence interval (low and high). Column 1 lists the NGC number, Cols. 2-4 the circular velocity at the last kinematic data point, Col. 5 gives the $M / L$ of the SC model for those galaxies for which this is within the confidence interval, Cols. 6-8 the central $M / L$ values, and Cols. 9-11 the $M / L$ ratios

\begin{tabular}{|c|c|c|c|c|c|c|c|c|c|c|}
\hline NGC & $\begin{array}{c}\text { low } \\
v_{\mathrm{c}}\left(R_{\max }\right) \\
\end{array}$ & $\begin{array}{c}\text { best } \\
v_{\mathrm{c}}\left(R_{\max }\right) \\
\end{array}$ & $\begin{array}{c}\text { high } \\
v_{\mathrm{c}}\left(R_{\max }\right) \\
\end{array}$ & $M / L_{\mathrm{SC}}$ & $\begin{array}{c}\text { low } \\
M / L_{\mathrm{B}}^{\mathrm{c}} \\
\end{array}$ & $\begin{array}{c}\text { best } \\
M / L_{\mathrm{B}}^{\mathrm{c}} \\
\end{array}$ & $\begin{array}{c}\text { high } \\
M / L_{\mathrm{B}}^{\mathrm{c}} \\
\end{array}$ & $\begin{array}{c}\text { low } \\
M / L_{\mathrm{B}} \text { ratio }\end{array}$ & $\begin{array}{c}\text { best } \\
M / L_{\mathrm{B}} \text { ratio }\end{array}$ & $\begin{array}{c}\text { high } \\
M / L_{\mathrm{B}} \text { ratio } \\
\end{array}$ \\
\hline 315 & 521.6 & 568.7 & 639.7 & & 11.0 & 10.5 & 9.6 & 1.468 & 1.830 & 2.533 \\
\hline 1399 & 373.8 & 424.1 & 466.1 & & 10.9 & 10.6 & 10.1 & 1.060 & 1.392 & 1.769 \\
\hline 2434 & 288.2 & 331.0 & 371.6 & & 6.2 & 6.0 & 6.4 & 1.417 & 1.918 & 2.253 \\
\hline 3193 & 283.9 & 303.0 & 334.7 & & 5.0 & 4.5 & 3.6 & 1.000 & 1.396 & 2.129 \\
\hline 3379 & 241.5 & 259.3 & 286.7 & & 4.7 & 4.5 & 4.2 & 1.000 & 1.219 & 1.602 \\
\hline 3640 & 270.8 & 279.2 & 288.2 & 3.7 & 3.6 & 3.7 & 3.7 & 1.004 & 1.030 & 1.097 \\
\hline 4168 & 264.7 & 286.8 & 306.2 & 6.5 & 6.1 & 5.8 & 5.4 & 1.058 & 1.295 & 1.602 \\
\hline 4278 & 398.2 & 415.9 & 424.4 & 8.8 & 8.6 & 7.6 & 6.4 & 1.005 & 1.244 & 1.544 \\
\hline 4374 & 380.8 & 409.7 & 441.8 & 9.5 & 8.7 & 8.8 & 8.3 & 1.023 & 1.168 & 1.457 \\
\hline 4472 & 429.9 & 463.7 & 502.0 & 7.8 & 6.7 & 7.0 & 6.5 & 1.022 & 1.134 & 1.450 \\
\hline 4486 & 491.9 & 506.5 & 566.9 & 11.8 & 10.9 & 9.4 & 8.1 & 1.044 & 1.280 & 1.853 \\
\hline 4486B & 233.9 & 248.7 & 268.5 & & 7.2 & 7.2 & 6.2 & 1.000 & 1.128 & 1.543 \\
\hline 4494 & 238.2 & 260.8 & 287.5 & 7.4 & 7.3 & 6.5 & 5.9 & 1.008 & 1.369 & 1.829 \\
\hline 4589 & 311.7 & 333.2 & 355.7 & 6.0 & 5.4 & 5.4 & 5.4 & 1.088 & 1.258 & 1.457 \\
\hline 4636 & 325.6 & 341.3 & 351.8 & 9.6 & 8.7 & 8.9 & 7.8 & 1.027 & 1.109 & 1.336 \\
\hline 5846 & 328.5 & 338.3 & 346.0 & & 10.1 & 9.7 & 9.5 & 1.053 & 1.164 & 1.244 \\
\hline 6703 & 190.5 & 221.8 & 248.4 & & 5.6 & 5.2 & 5.0 & 1.000 & 1.452 & 1.885 \\
\hline 7145 & 193.0 & 210.2 & 254.5 & & 3.5 & 3.9 & 3.7 & 1.922 & 2.026 & 3.162 \\
\hline 7192 & 256.6 & 269.9 & 292.0 & & 5.1 & 4.6 & 4.7 & 1.332 & 1.643 & 1.876 \\
\hline 7507 & 350.6 & 399.0 & 471.8 & & 6.2 & 5.9 & 5.9 & 1.417 & 1.937 & 2.700 \\
\hline 7626 & 372.9 & 401.3 & 437.5 & & 9.0 & 8.1 & 7.8 & 1.406 & 1.814 & 2.223 \\
\hline
\end{tabular}

Table 8. Comparison with previous mass determinations. Columns 2 and 3 show the newly determined mass-to-light ratios at the innermost (Col. 2) and outermost (Col. 3) kinematic data points and at the half-luminosity radius (Col. 4) determined by Saglia et al. (1992), from the respective "best" model, for part of the sample galaxies. Previous $M / L$ values converted to the $B$-band and to the same galaxy distances are listed from van der Marel (1991; Col. 5), Lauer (1985; Col. 6), Saglia et al. (1992, luminous component, Col. 7, luminous plus dark component at the half-luminosity radius determined by the fit, Col. 8)

\begin{tabular}{|c|c|c|c|c|c|c|c|}
\hline NGC & $M / L_{\mathrm{cent}}$ & $M / L_{\max }$ & $M / L_{1 / 2}$ & $\begin{array}{r}M / L_{\mathrm{B}} \\
\mathrm{vdM}\end{array}$ & $\begin{array}{c}M / L_{\mathrm{B}} \\
\text { Lauer }\end{array}$ & $\begin{array}{r}M_{\mathrm{L}} / L \\
\text { SBS }\end{array}$ & $\begin{array}{r}M / L_{1 / 2} \\
\text { SBS }\end{array}$ \\
\hline 1399 & 10.6 & 14.7 & 15.5 & 8.8 & - & 10.2 & 16.4 \\
\hline 3379 & 4.5 & 5.5 & 5.1 & 4.7 & 8.3 & 6.0 & 10.2 \\
\hline 4278 & 7.6 & 9.5 & 12.5 & - & - & 8.8 & 11.1 \\
\hline 4374 & 8.8 & 10.3 & 11.1 & 9.7 & 9.4 & 7.0 & 17.5 \\
\hline 4472 & 7.0 & 8.0 & 11.9 & 8.0 & 10.9 & 6.3 & 9.6 \\
\hline 4486 & 9.4 & 12.0 & 26.1 & 10.2 & - & 7.5 & 11.3 \\
\hline 4494 & 6.5 & 8.9 & - & 11.6 & - & - & - \\
\hline 4636 & 8.9 & 9.8 & 17.6 & 9.1 & 13.3 & 10.5 & 14.4 \\
\hline 5846 & 9.7 & 11.3 & - & 10.7 & 12.7 & - & - \\
\hline 6703 & 5.2 & 7.6 & - & - & 6.4 & - & - \\
\hline 7145 & 3.9 & 8.0 & - & 6.4 & - & - & - \\
\hline 7507 & 5.9 & 11.3 & 10.1 & 5.5 & - & $5.2^{1}$ & $11.3^{1}$ \\
\hline 7626 & 8.1 & 14.7 & 12.7 & 8.3 & 10.3 & 7.7 & 10.5 \\
\hline
\end{tabular}

(1) From Bertin et al. (1994) using the same modelling. 

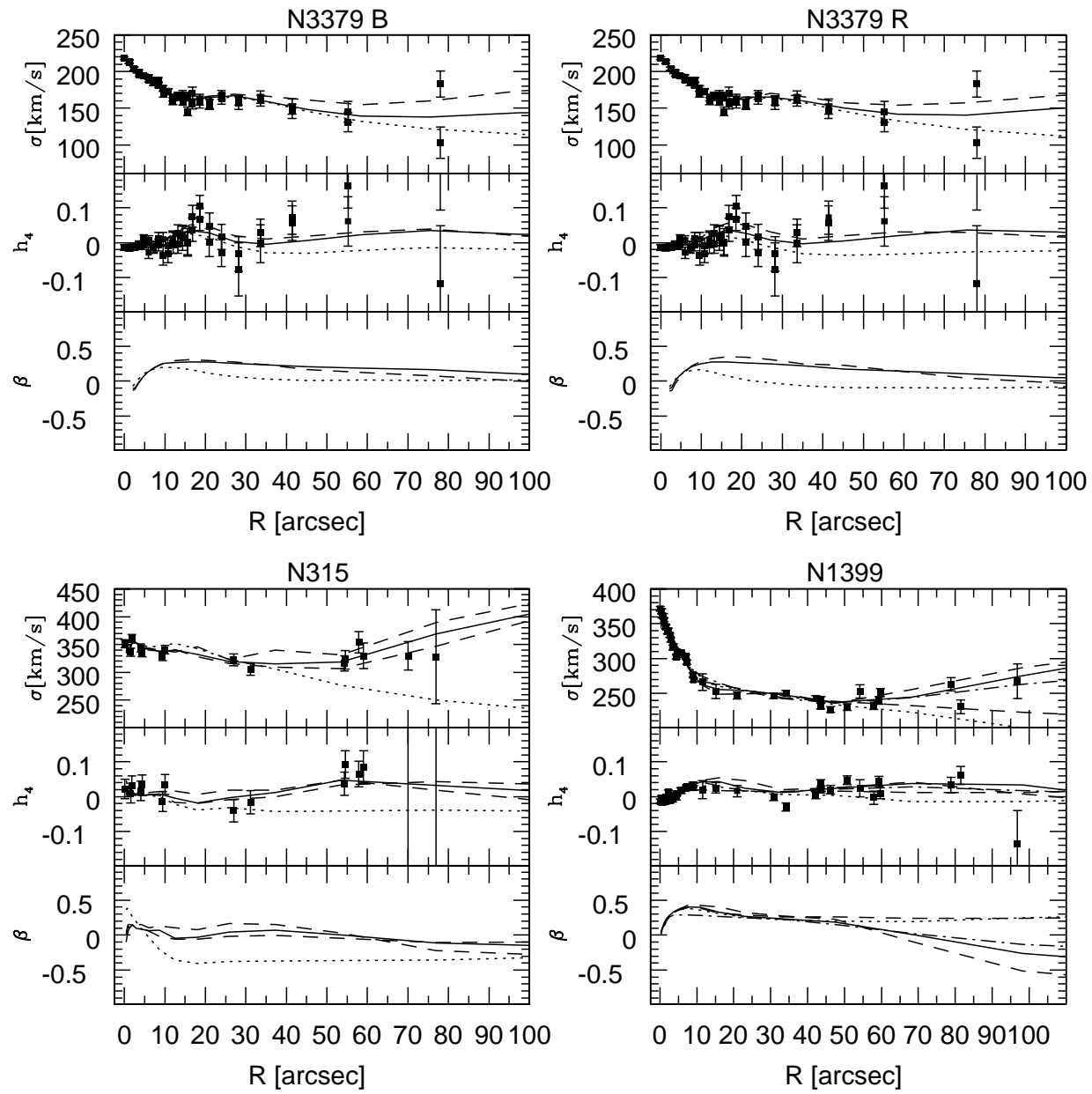

Fig. 12. Fits to the kinematic data of NGC 3379 using $B$-band photometry (top left), NGC 3379 again but using $R$-band photometry (top right), NGC 315 (bottom left), and NGC 1399 (bottom right). In each panel the uppermost of the three plots shows the velocity dispersion, the middle plot the $h_{4}$ parameter, and the lower plot the intrinisic velocity anisotropy parameter $\beta$. Note that the velocity dispersion scale changes between different galaxies. For each galaxy we show a "best" halo model from the middle of the confidence range (full line), two models from the boundary of the confidence interval (dashed lines), and the self-consistent model with constant $M / L$ (dotted line). When only one dashed line is shown, the lower boundary of the confidence interval is represented by the constant $M / L$ model. For NGC 1399 we show an additional model (long dash - dotted line), constructed from a very different set of basis functions in the same "best" halo potential; this shows that the type of basis used does not significantly influence the derived kinematics (see also Sect. 5.3)

used in the present paper. The three $M / L_{\mathrm{B}}$ values given for each galaxy from our analysis are the central value of the respective "best" model, corresponding to the "maximal stellar mass" $M / L_{\mathrm{B}}$ of the stellar population, the "best" cumulative $M / L_{\mathrm{B}}$ inside the last kinematic data point, and the "best" cumulative $M / L_{\mathrm{B}}$ inside the halfluminosity radii determined by Saglia et al. (1992). The latter two values thus refer to the total mass (luminous and dark) out to these radii.

Comparing our central values with those of van der Marel (1991) shows that the new mass-to-light ratios are similar in the mean with individual differences generally less than $20 \%$ and particularly large discrepancies of $\sim 50 \%$ for NGC 7145 and by a factor of two for NGC 4494. The latter galaxy was off in all of van der
Marel's correlation plots. van der Marel used axisymmetric two-integral models but did not take into account lineprofile shape information. Because these models have the property of flattening the mass distribution by equatorial near-circular orbits (Dehnen \& Gerhard 1993), they generally predicted too much motion on the major axis and thus van der Marel's $M / L$ values were taken to be weighted averages of the minor and major axis fits.

The mass-to-light ratios given by Lauer (1985) are estimates based on the standard King formula for isotropic cores, and are always higher than our new determinations. Differences less than $20 \%$ are observed between our central $M / L$ values and those for the luminous component of Saglia et al. (1992). The $M / L$ ratios computed at the half-luminosity radii used by Saglia et al. (1992) also agree 

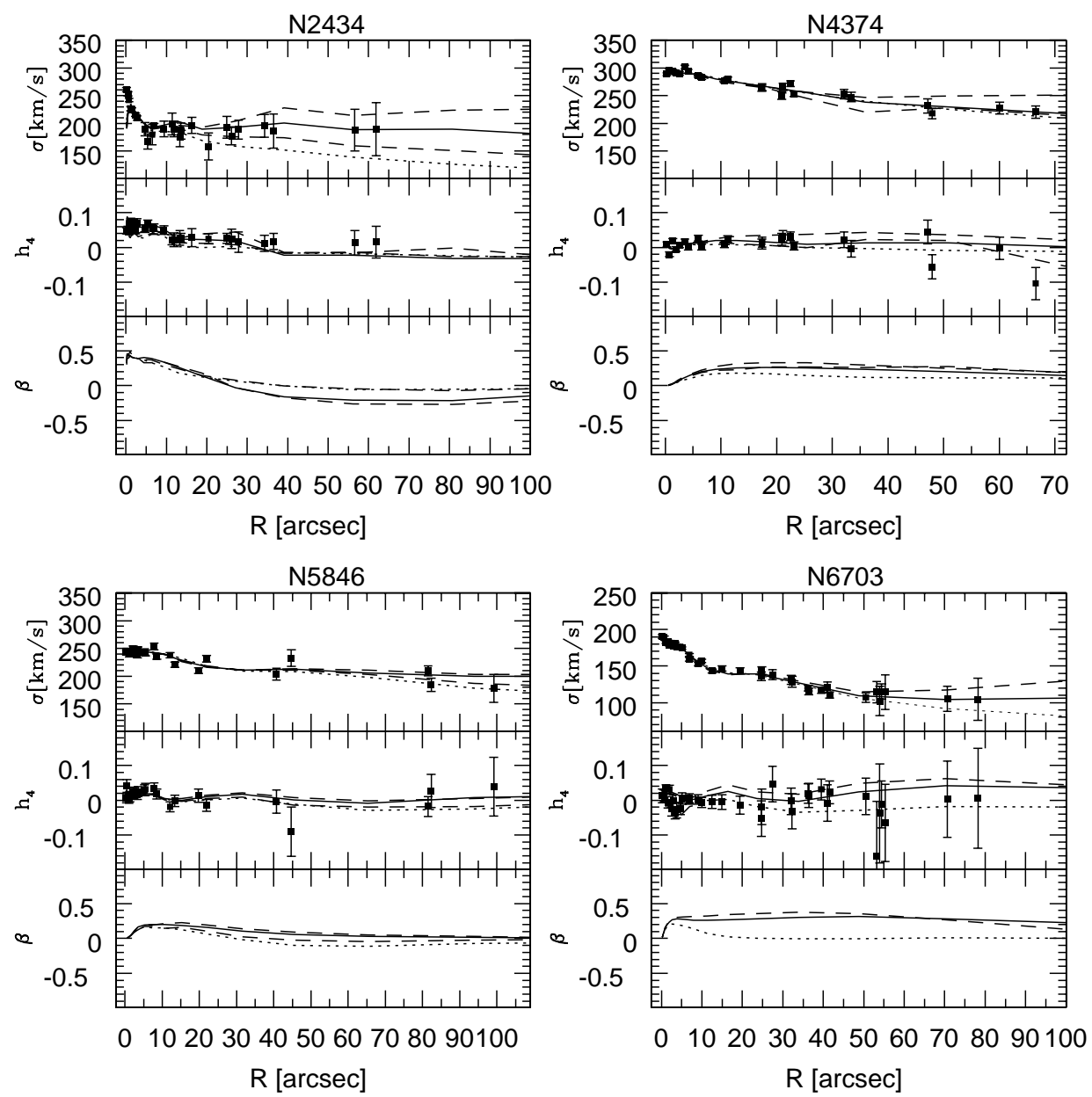

Fig. 13. As in Fig. 12 for NGC 2434 (top left), NGC 4374 (top right), NGC 5846 (bottom right) and NGC 6703 (bottom right)

reasonably well. However, for NGC 3379 and NGC 4374 Saglia et al. (1992) fit a rather massive dark halo not detected here, while for NGC 4486 we find a rather large extrapolated $M / L$ also seen in the X-ray data.

For a few of our sample galaxies other data are available besides the absorption line kinematics, which considerately strengthen the evidence for dark halos in some of these galaxies. Several galaxies have velocity measurements of "discrete tracers" (planetary nebulae, PNe; globular clusters, GC; dwarf galaxies bound to the respective galaxy, D) at intermediate to large radii; see Table 9 for a list. In these cases we performed a maximum likelihood analysis along the lines of $\mathrm{S}+2000$ to test the consistency of our mass models with these data and select the most probable ones. Results are shown in Figs. 24, 25, and 27 and are discussed in the individual galaxy subsections below. Mass-profiles derived from X-ray data are still rare. S+2000 compared their models for NGC 1399 with massprofiles derived from ASCA measurements by Ikebe et al. (1996). For three further galaxies of our sample, such profiles are also available: Besides the two brightest ellipticals of the Virgo-cluster, NGC 4486 and NGC 4472, for which
ROSAT data were analysed by Schindler et al. (1999), NGC 4636 has an X-ray mass profile from ASCA-data (Matsushita et al. 1998). For these three galaxies we compare the mass profiles of our absorption-line models to the X-ray mass profiles: NGC 4486 is shown in Fig. 28, NGC 4472 in Fig. 26 and NGC 4636 in Fig. 29. See the notes on individual galaxies below.

\subsection{Estimate of uncertainties due to rotation and non-sphericity}

A number of galaxies in our sample show slight rotation, and some may be flattened and seen along special viewing directions such as to appear round on the sky. Since our modelling assumes spherical symmetry and no rotation, we need to assess the uncertainties in the results associated with these assumptions.

The most rapidly rotating galaxy in this sample is NGC 3379, which reaches a maximum $(v / \sigma)_{\max } \simeq 0.3$. In the modelling reported above, we have simply ignored $v$ and $h_{3}$. As an alternative, we have fitted an even velocity distribution to the spectral line shapes, forcing 

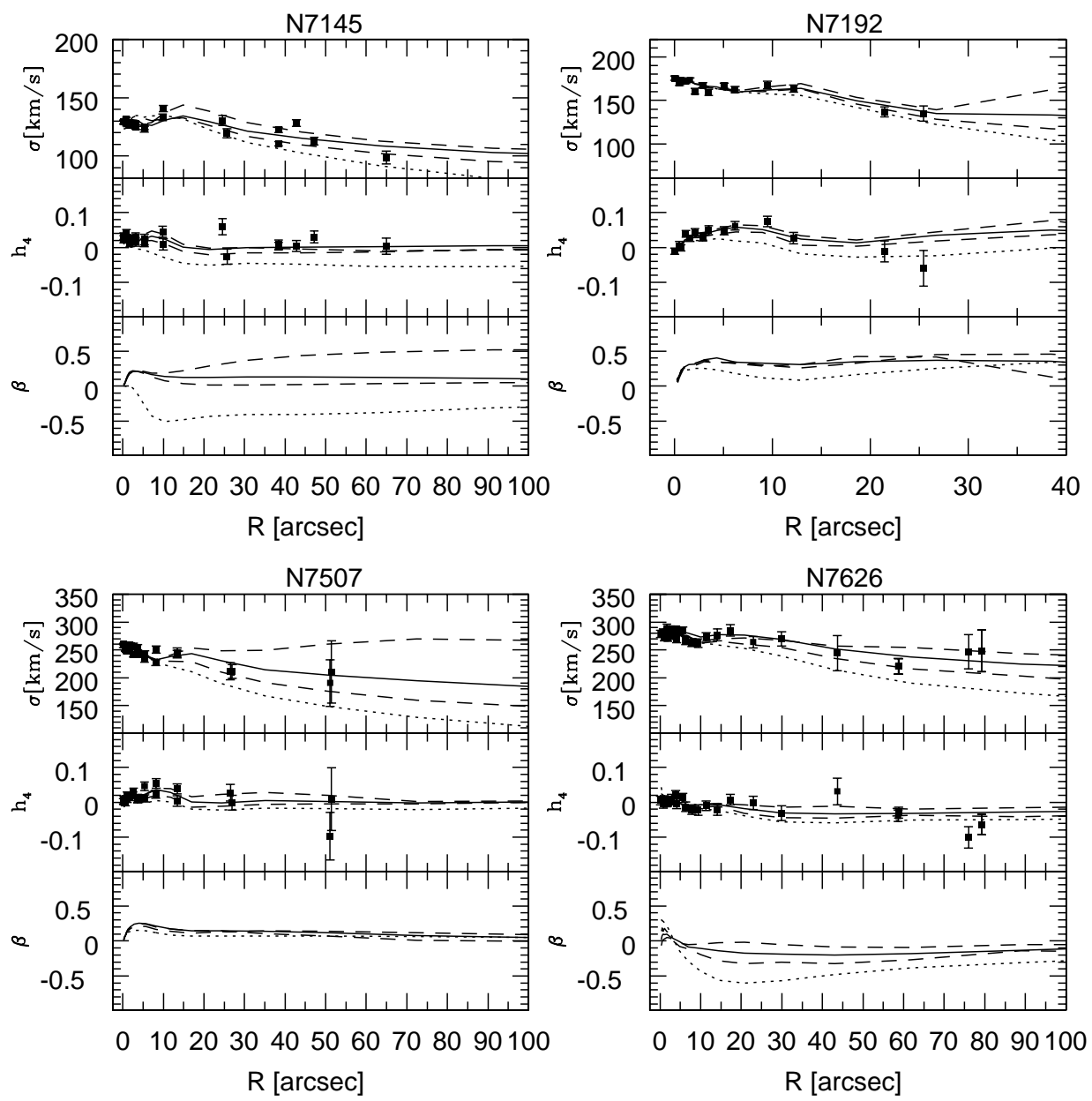

Fig. 14. As in Fig. 12 for NGC 7145 (top left), NGC 7192 (top right), NGC 7507 (bottom left) and NGC 7626 (bottom right)

$v=h_{3}=0$, and have then tested the effect this has on the modelling results. In this case, the velocity dispersions near maximum rotation are larger by about $10 \%$, while the $h_{4}$ values become slightly more negative. From simple arguments we would have expected an increase in $\sigma$ by $\simeq\left(1+0.3^{2}\right)^{1 / 2}$, i.e., about $5 \%$. In these kinematics derived from symmetrized line profiles there are significant systematic deviations between both sides of the galaxy.

Modelling these data as before, with the same regularization parameter, gives the following results. The anisotropy profile remains similar. The mass scale increases slightly; in the model without halo, the mass-tolight ratio thus increases to $M / L=5.44$ (previously 4.7 ), while the central value in the best-fit halo potential is now $M / L=5.27$ (previously 4.5). In the three-integral models of Gebhardt et al. (2000) the stellar mass-to-light ratio is in the range $4.94-5.51$ when corrected to $B$-band (using $B-V=0.94$ from the profiles) and to our distance (13.2 instead of $10.4 \mathrm{Mpc}$ ). For NGC 3379, the uncertainty in $M / L$ due to the neglect of rotation is therefore $\sim 15 \%$. If we scale from these results, in the next most rapidly rotating galaxies in the sample, with $(v / \sigma)_{\max } \simeq 0.15$, we
Table 9. Galaxies with kinematics for discrete tracer populations. The number of PNe (planetary nebulae), GC (globular clusters), and D dwarf galaxy velocities is listed for each galaxy. Both for NGC 4472 and NGC 4486 there are 5 confirmed dwarf galaxy companions and 3 further candidates. In these cases all 8 velocities were used for calculating the velocity dispersion. The 20 dwarfs around NGC 5846 (Zabludoff \& Mulchaey 1998) lie too far from the galaxy to constrain our models

\begin{tabular}{lr}
\hline & \\
NGC & Sort \\
\hline & \\
1399 & $37 \mathrm{PN}, 74 \mathrm{GC}$ \\
3379 & $29 \mathrm{PN}$ \\
4472 & $57 \mathrm{GC}, 8(5) \mathrm{D}$ \\
4486 & $224 \mathrm{GC}, 8(5) \mathrm{D}$ \\
5846 & $20 \mathrm{D}$ \\
\hline
\end{tabular}

will underestimate the rms velocity $\sigma$ by $\simeq 3 \%$ and the mass-to-light ratio by $5 \%$. For $(v / \sigma)_{\max } \simeq 0.1$ the corresponding errors will be negligible.

We now turn to the effects of a possible flattening along the line-of-sight. From the results of Tremblay \& Merritt 

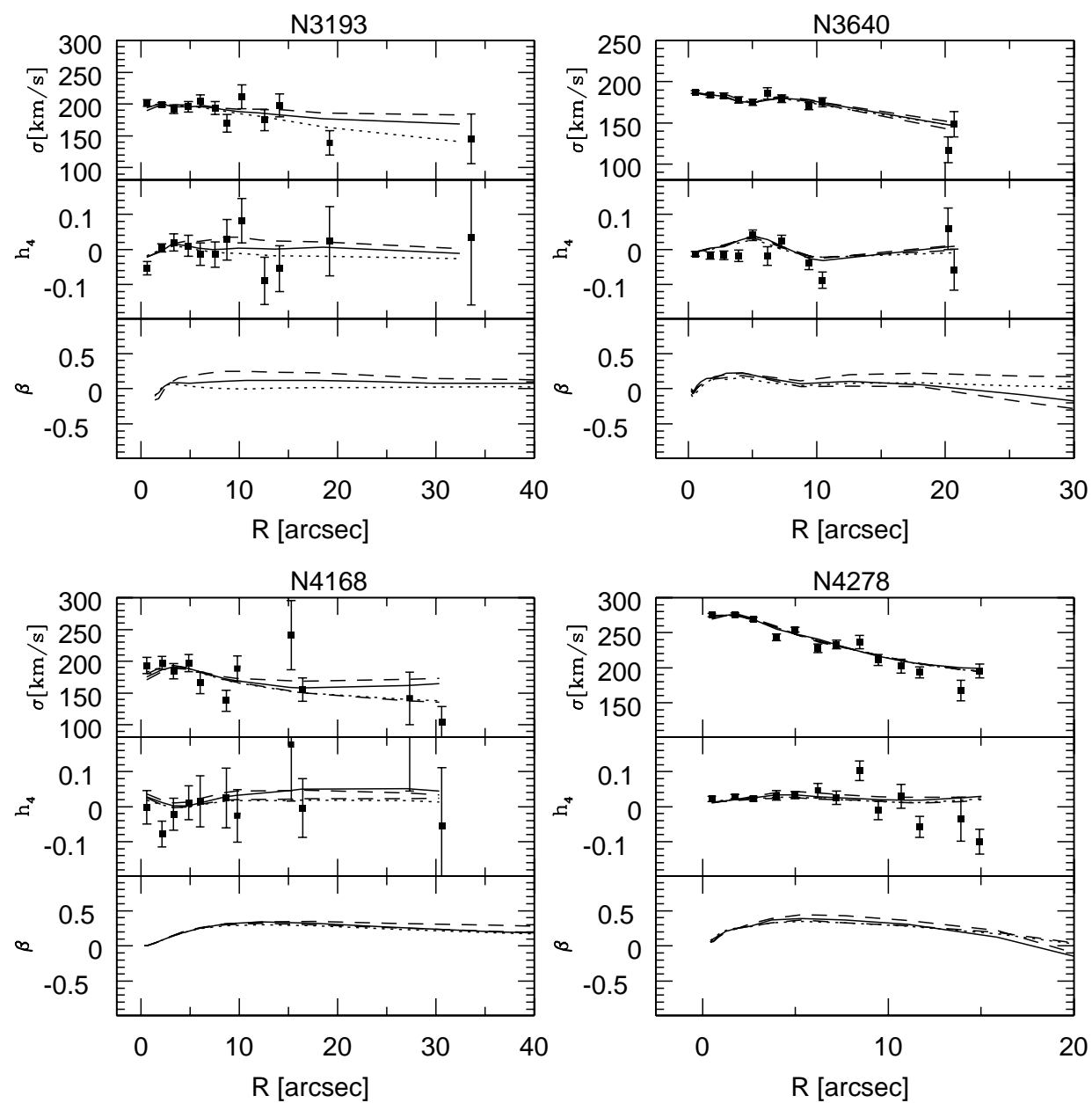

Fig. 15. As in Fig. 12 for NGC 3193 (top left), NGC 3640 (top right), NGC 4168 (bottom left) and NGC 4278 (bottom right)

(1996), who modelled the apparent shape distribution of a large sample of ellipticals, we can estimate the typical intrinsic flattening of our sample galaxies. These are bright elliptical galaxies: the large majority falls into the highest luminosity bin of Tremblay \& Merritt, even after correcting for the difference in assumed distance. Taking their result for a distribution of triaxial shapes with constant triaxiality parameter $(1-b / a) /(1-c / a)=0.3$, and approximating their derived distribution of $c / a$ in the bottom panel of their Fig. 4 by a Gaussian, we find that the mean intrinsic short-to-long axis ratio $c / a$ of all luminous galaxies that project to E1 (0.9) or rounder is 0.79 . (The corresponding value for the faint ellipticals with $M_{\mathrm{B}}=-18.5$, assuming oblate symmetry and using Fig. 3 of Tremblay $\&$ Merritt, would be 0.73.) The mean flattening along the line of sight is then somewhat smaller, because not all galaxies that project to E1 or rounder have their short axes along the line-of-sight.

If an apparently round galaxy is flattened along the line-of-sight, this will have the following effects on our results. (i) The line-of-sight velocity dispersion may be less than the dispersion in the plane of the sky if the sys- tem is flattened by anisotropy, and it will also not measure any kinetic energy corresponding to rotation $\bar{v}$ in the plane of the sky. This leads to an underestimate of the mass-to-light ratio. We can estimate the magnitude of this effect from the tensor virial theorem, in the form $\left(\bar{v}^{2}+2 \sigma_{x}^{2}\right) / \sigma_{z}^{2} \simeq 2(a / c)^{0.9}$ (Gerhard 1994, Eq. 5.10). For a mean $c / a=0.8$ the total kinetic energy thus increases by a factor 1.15 , for constant $M / L$. At the same time the flattened potential well is deeper for fixed mass and $M / L$; using relations from Binney \& Tremaine (1987, Sect. 2.5) the total potential energy increases by a factor 1.07 . Since the former is linearly proportional to $M / L$ and the latter scales like $(M / L)^{2}$, reestablishing virial equilibrium implies an increase in $M / L$ by about $8 \%$. (For a galaxy with intrinsic $c / a=0.6$, the corresponding value would be $21 \%$.)

(ii) A more indirect effect is through the shape of the absorption line profiles. This depends critically on the stellar distribution function and hence the mechanism through which the galaxy is flattened (see Dehnen \& Gerhard 1993). If the galaxy is flattened by equatorial near-circular orbits, as is the case in two-integral models 

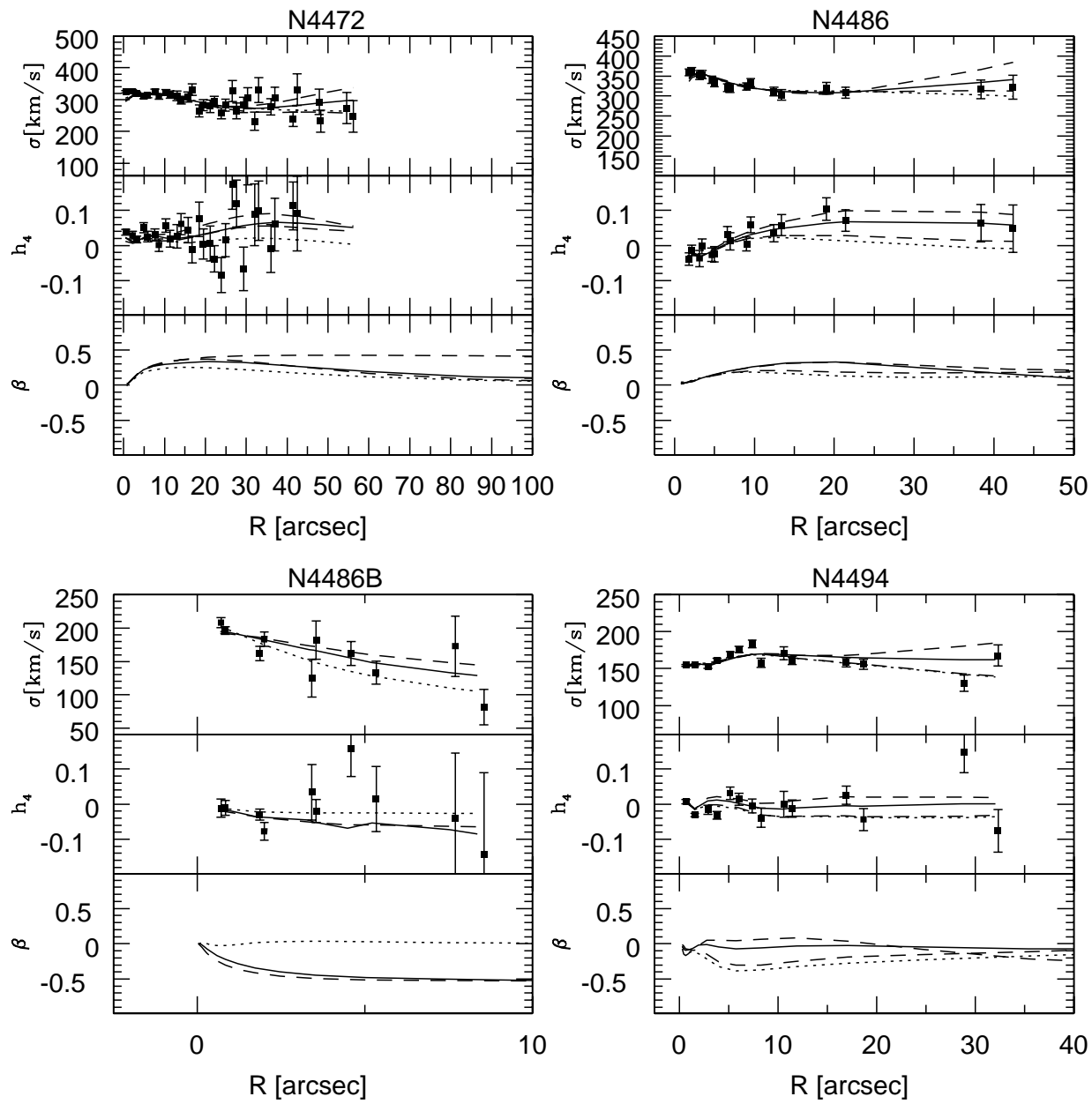

Fig. 16. As in Fig. 12 for NGC 4472 (top left), NGC 4486 (top right), NGC 4486B (bottom left) and NGC 4494 (bottom right)
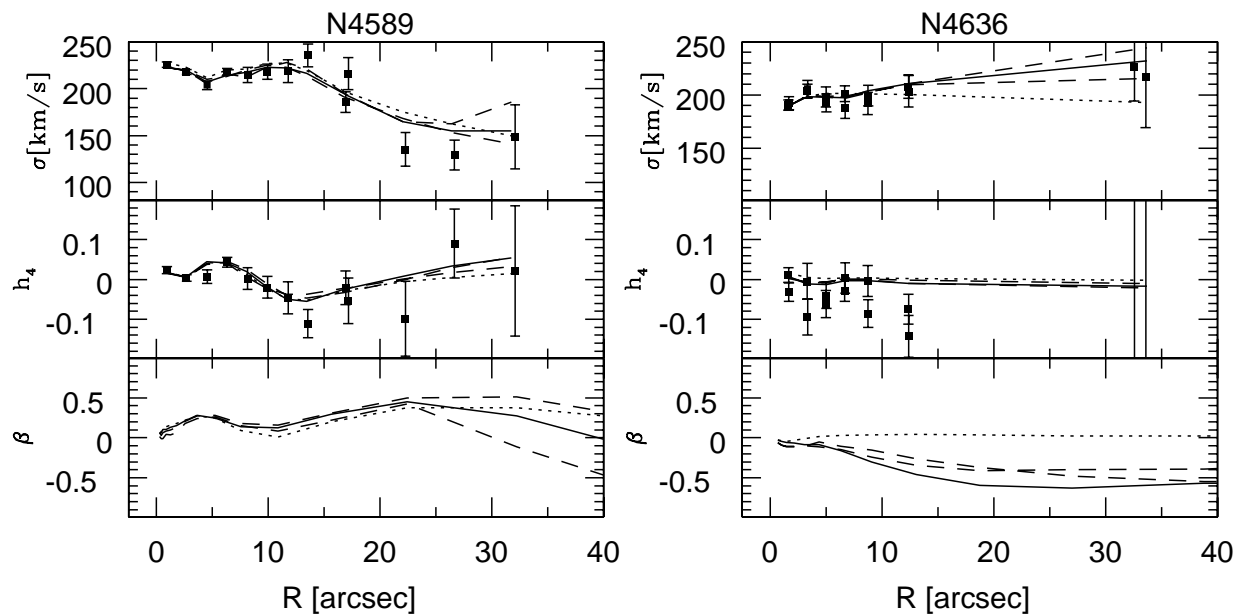

Fig. 17. As in Fig. 12 for NGC 4589 (top left), and NGC 4636 (top right) 


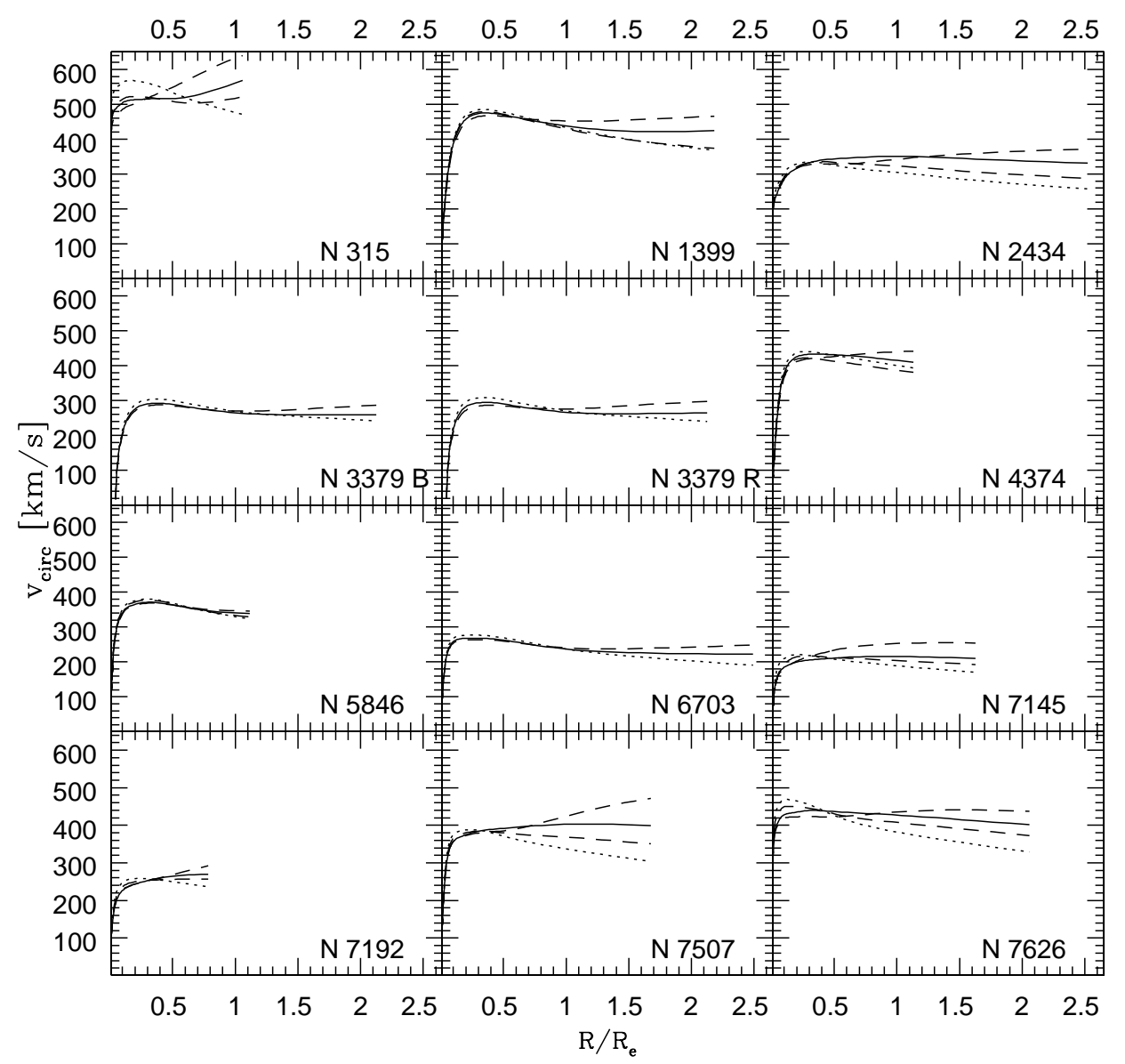

Fig. 18. Inferred circular velocity curves, plotted out to the radius of the last kinematic data point. The "best" model from the middle of the confidence range is shown as the solid line. The two dashed lines show models at the boundaries of the $95 \%$ confidence interval and mark the implied uncertainty within our range of halo models. The self-consistent (SC) model with only luminous mass is shown as the dotted line. In cases where the SC model is the one at the low $v_{\mathrm{c}}$ end of the confidence interval, the evidence for extra dark mass is less than $2 \sigma$ and only three lines are shown

$f\left(E, L_{z}\right)$, then these orbits seen face-on near $v \simeq 0$ generate a more peaked line profile with larger $h_{4}$, which could mimic some radial anisotropy when interpreted through a spherical model. This effect was investigated by Dehnen \& Gerhard (1994) and by Magorrian (2000). From Fig. 12 of Dehnen \& Gerhard (1994) for a de Vaucouleurs-like luminosity model inside $\sim R_{\mathrm{e}}$, the increase in $h_{4}$ from $c / a=1$ to $c / a=0.8$ is $\simeq 0.01$, the density profile being still relatively shallow there $(\mathrm{d} \log \rho / \mathrm{d} \log r \gtrsim-2)$. There is a simultaneous decrease in the velocity dispersion by about $3-4 \%$, which will cause another upward correction of $M / L$. The implied $\Delta h_{4}$ is significantly smaller than the $h_{4}$ values responsible for the radial anisotropy in our models. In addition, because most of our galaxies are luminous ellipticals, they are not well-described by twointegral models (e.g., van der Marel 1991), and for other flattening mechanisms the effect on the line profile shapes will be smaller.

More significant could be the effects of hidden, sufficiently luminous and cold face-on disks, which might lead to a larger increase in $h_{4}$. Using Fig. 1 of BSG94, we estimate that such a disk of disk-to-bulge ratio $\epsilon$ would generate $h_{4} \approx 0.5 \epsilon$, as a compromise between the increased flux at zero velocities due to the disk and the lack of effect on the large velocity tail of the profile also measured by $h_{4}$. The histogram of disk-to-bulge ratios for the known kinematically decoupled disks or toruslike structures (Bender \& Saglia 1999) shows that for boxy elliptical galaxies $\epsilon \lesssim 0.02$ and for disky ellipticals $\epsilon \lesssim 0.1$, with some cases (NGC 4660) with $\epsilon$ up to 0.3 . Generally these components are fairly small, extending to typical radii $r_{\mathrm{b}} / R_{\mathrm{e}} \approx 0.1-0.3$ (Mehlert et al. 1998). They can be detected photometrically only if seen nearly edge-on, but might be present in virtually all ellipticals. The three galaxies of our sample appearing in Mehlert et al., NGC 4472, NGC 4494, NGC 7626, have $r_{\mathrm{b}}=$ $6,7,8$ arcsec, respectively. Therefore we conclude that the anisotropy profiles should not suffer from the possible presence of disks at radii $\gtrsim 0.2 r_{\mathrm{e}}$ but might overestimate 


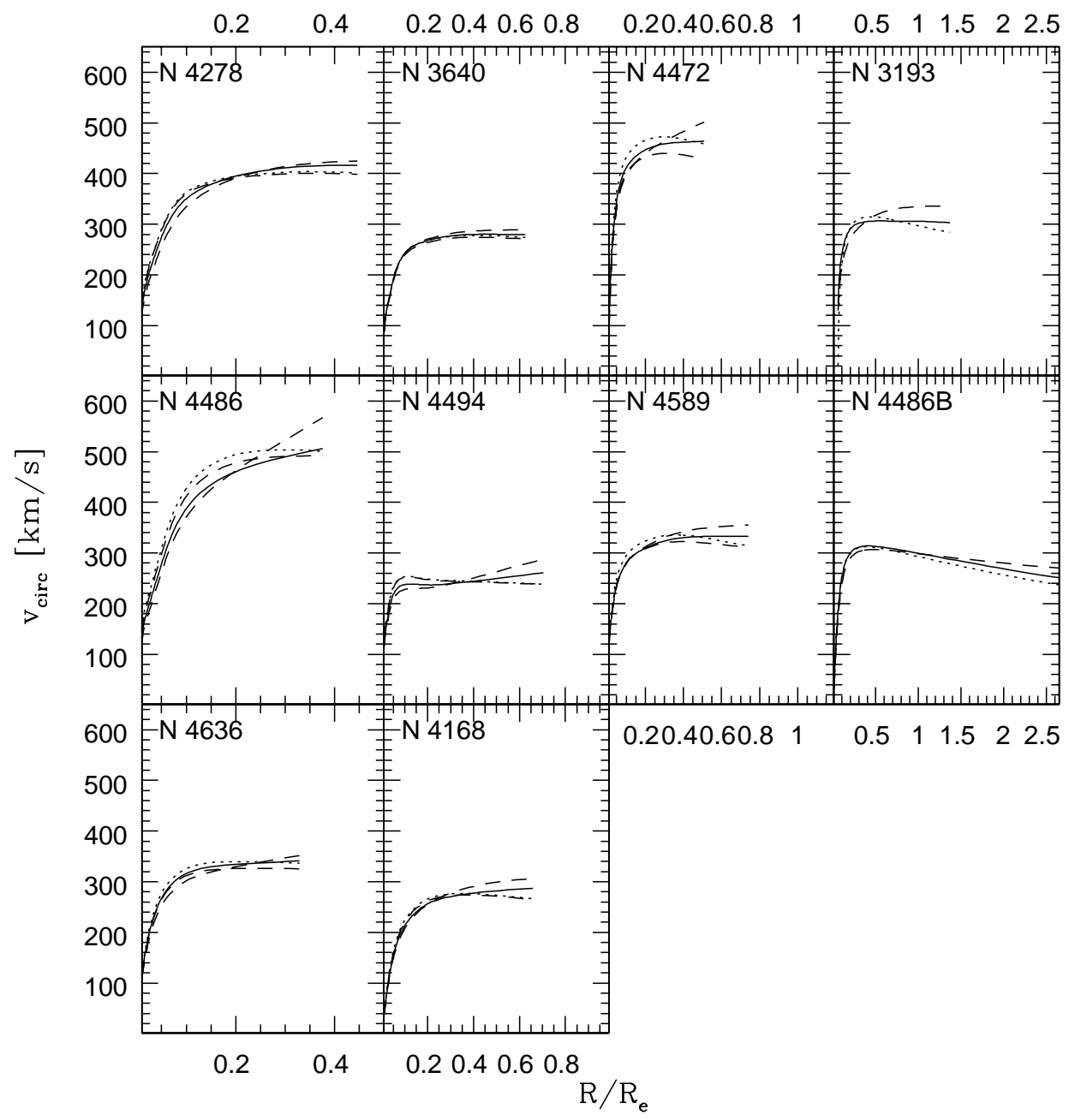

Fig. 19. Circular velocity curves for the sample with kinematic data from BSG94, as in Fig. 18

the real anisotropy in the inner regions of the galaxies by $\approx 0.2$.

Finally, we note that the analysis of the two flattened, non-rotating elliptical galaxies NGC 1600 (E3.5, near edge-on) and NGC 2300 (E2, edge-on or inclined) with three-integral models by Matthias \& Gerhard (1999) and Kaeppeli (1999) resulted in similar anisotropy profiles to those we have obtained in this paper. As a further test, we have for these two galaxies taken the major axis photometric profile and major axis kinematics and applied our spherical modelling to these data, ignoring the flattening. The resulting models slightly underestimate the radial anisotropy as compared to the edge-on three-integral models, and the $V$-band $M / L_{V}$ come out 5.5 and 6.5 , respectively, compared to 6.0 and 6.25 in the axisymmetric analysis, a $5-10 \%$ difference.

\section{2. $N G C 315$}

This galaxy has a shallow luminosity profile and considerable structure in the kinematic profiles. In the analy- sis the errors of the two outermost $h_{4}$ data points were set to unity to make sure that they do not influence the fit. These points are uncertain due to low signal-to-noise ratio. The dip in the velocity dispersion profile together with the clustering of the data points and the substantial scatter between the inner data points as compared to the kinematic errors (Table 5) make this dataset difficult to model. We obtained more regular results with the second method of treating the presumably systematic errors (see Sect. 4); the models shown in Figs. 12, 18 and 20 are obtained with this method. The $95 \%$ confidence line is then at $\chi^{2}=1.2$.

Our simple halo models appear to be not very well suited for this galaxy. Together with the shallow distribution of the luminous mass these halo potentials favour rising velocity dispersion profiles in the outer parts when the inner data are well-fitted. There is little evidence in the data for this. Therefore while the upper boundary of the confidence interval appears reliable from our model results, the lower boundary should probably include models with a flat velocity curve with 


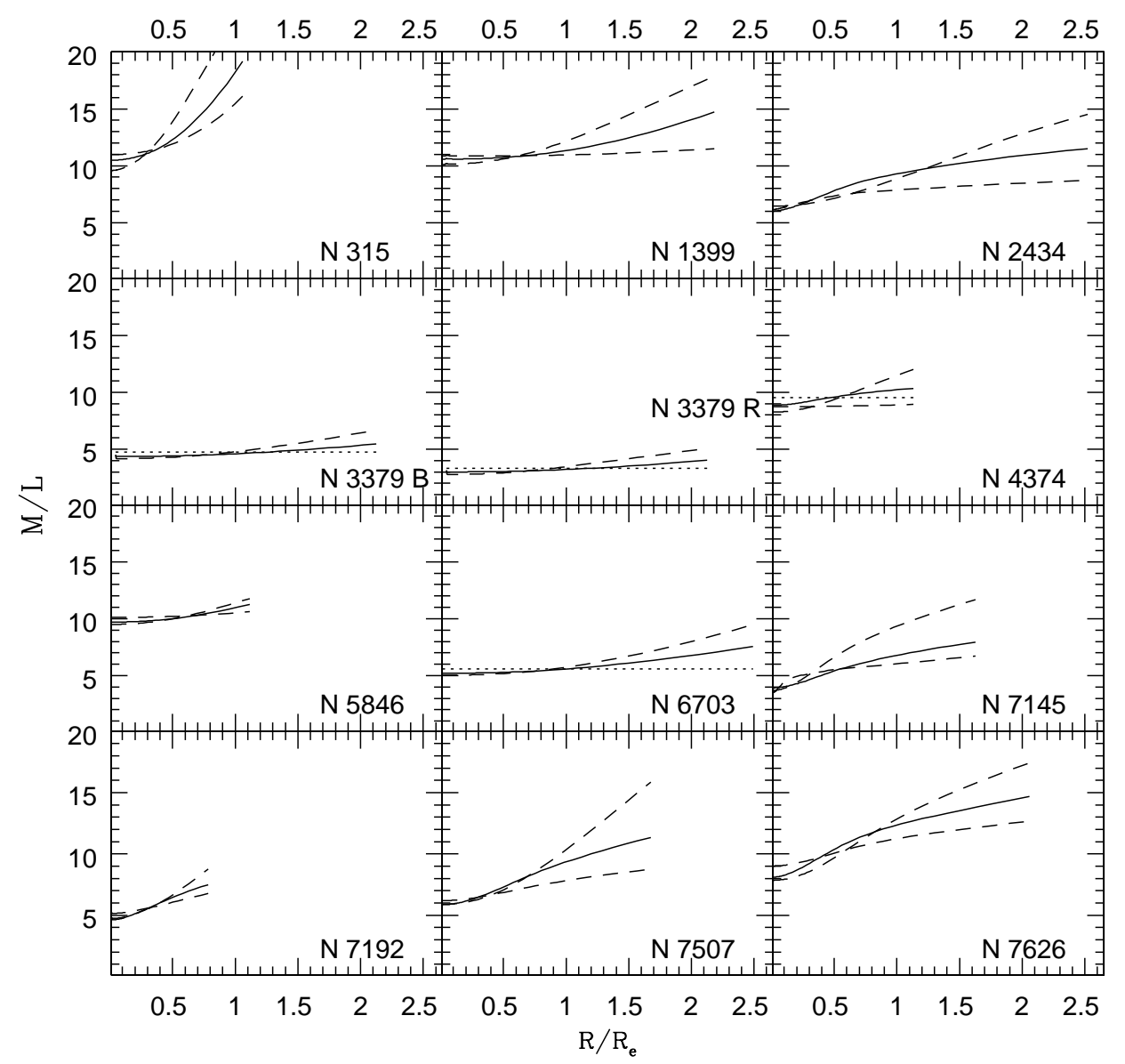

Fig. 20. Inferred mass-to-light ratios in the $B$-band as function of radius. For NGC 3379 we also show the corresponding $R$-band plot. The "best" model from the middle of the confidence range is shown as the solid line. The two dashed lines show models at the boundaries of the $95 \%$ confidence interval and mark the implied uncertainty within our range of halo models. When the confidence interval includes the self-consistent model with constant $M / L$, this is shown as the dotted line. In some cases it is identical with the lower-mass confidence boundary and then replaces the lower dashed line. In other cases it may have slightly more mass than the lower boundary halo model because of a different velocity scaling resulting from the fit to the kinematic data. In such cases the evidence for extra dark mass is less than $2 \sigma$

$v_{\mathrm{c}} \simeq 520 \mathrm{kms}^{-1}$. Nonetheless, the self-consistent model falls short of fitting the data from about $40^{\prime \prime}$.

\section{3. $N G C 1399$}

This galaxy is modelled in detail by $\mathrm{S}+2000$. As described there the line-of-sight velocities of 37 PNe from Arnaboldi et al. (1994) and 74 GCs from Kissler-Patig et al. (1998) favour the high-mass halo models in Fig. 18. These are also consistent with X-ray mass profiles from Ikebe et al. (1996). The abrupt increase of the dispersion profile seen in the PNe and GCs just beyond the end of the absorption line data needs to be confirmed with larger PNe samples.

Since the absorption line data only weakly imply dark matter in this galaxy, we have used NGC 1399 to show that the details of how to determine the $95 \%$ confidence line do not significantly influence the result; see Sect. 4 and Fig. 22.
Finally we have used this galaxy to check that within the uncertainties the derived kinematic properties are independent of the choice of basis functions employed in the modelling. The long dashed - dotted lines in the panel for NGC 1399 in Fig. 12 show the fit to the kinematic data using a distribution function composed from a basis of 39 radially anisotropic plus the isotropic basis functions in the same best-fit halo potential as for the full line with the basis used in $\mathrm{S}+2000$, which contained 55 tangentially anisotropic, one radially anisotropic, and the isotropic models. Notice the close similarity of these two models in both the velocity dispersion and $h_{4}$ profiles, although the new model has a significantly higher $\chi^{2}$. Both models also agree in the shape of the derived anisotropy profile, showing a similar radially anisotropy main body between $5^{\prime \prime}-50^{\prime \prime}$ and turning towards a more isotropic structure both in the centre and in the outer parts. The most significant difference is the slightly lower maximum anisotropy resulting with the radial basis. 


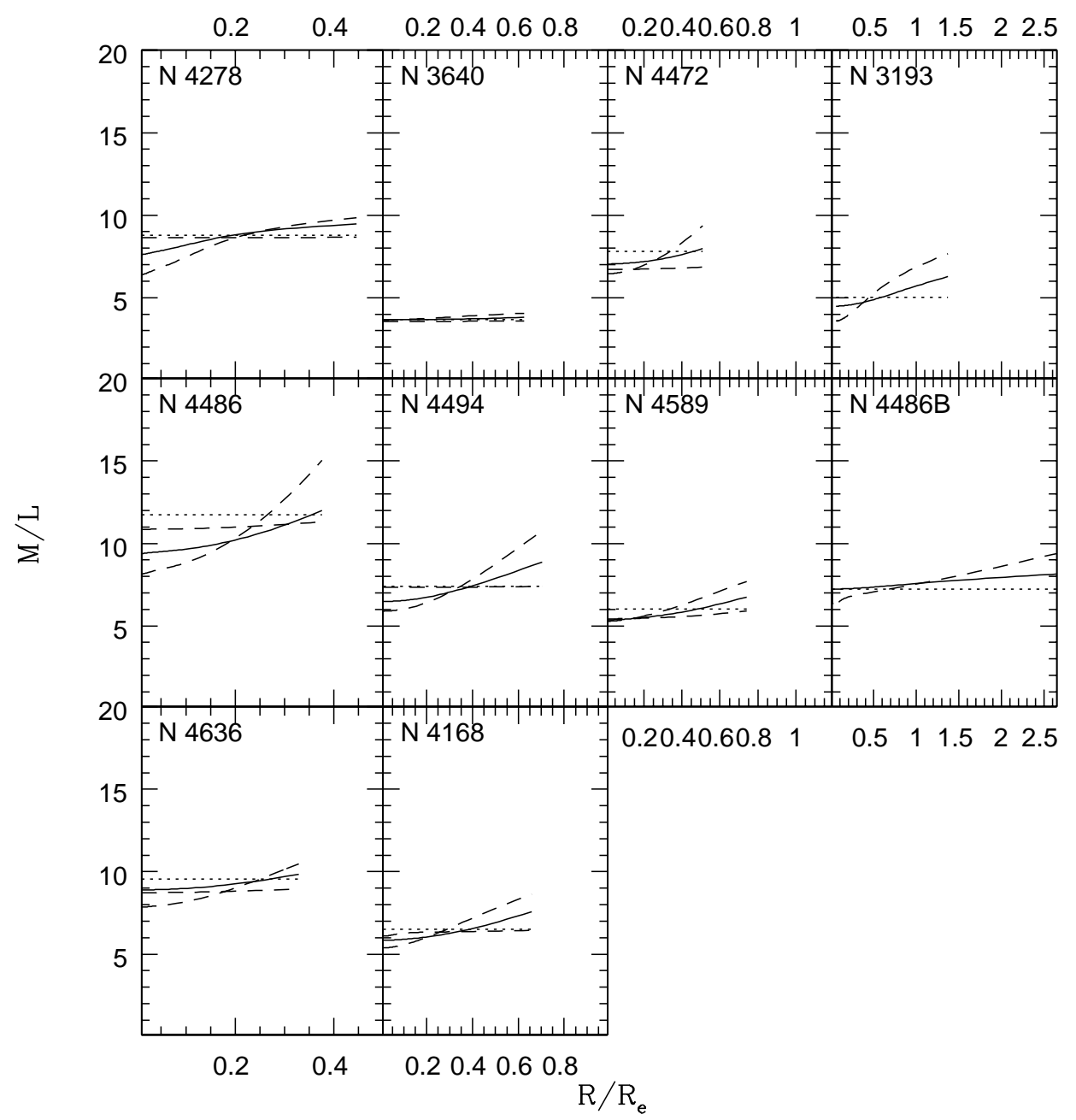

Fig. 21. Same as Fig. 20 for the sample of galaxies with kinematics from BSG94

This agreement in the inherently less accurate intrinsic quantity $\beta$ is gratifying.

\subsection{NGC 2434}

NGC 2434 was modelled extensively by Rix et al. (1997) with a modified Schwarzschild method. We have used the same photometric and kinematic data from Carollo \& Danziger (1994), but have ignored the kinematic data inside $R<1$ arcsec for the fit. The steep increase in $\sigma$ and the relatively high values of $h_{4}$ may indicate a central dark mass.

The results for this galaxy allow a direct comparison of both methods. In both analyses the SC models are ruled out by a large margin in $\chi^{2}$. Also, the circular velocity curves of the respective best models are very similar (see Figs. 13 and 8 of Rix et al. 1997). Our value of the central $M / L_{\mathrm{B}}=6.00 \pm 0.3$ whereas Rix et al. obtained $M / L_{\mathrm{B}}= \pm 3.9 \pm 0.35$, probably because their different halo models contributed more dark mass near the center. Our "best" value for the circular velocity at the outermost kinematic data point $\left(331 \mathrm{~km} \mathrm{~s}^{-1}\right.$ at $\left.62^{\prime \prime}\right)$ agrees well with their best-fitting models. Another difference concerns the intrinsic velocity anisotropy. Rix et al. found an almost constant anisotropy of $\beta \simeq 0.5$ which is slightly more than our maximum value at $\simeq 0.5 R_{\mathrm{e}}$. Outwards of 10 arcsec our anisotropy falls, reaching a value of zero at 40 arcsec (roughly $2 R_{\mathrm{e}}$ ) from whereon outwards it is consistent with isotropic. Comparing both figures suggests that Rix et al. may slightly overestimate the outer anisotropy while our model may slightly underestimate it; so both results may not be inconsistent, emphasizing the larger uncertainties in any derived intrinsic quantities.

\section{5. $N G C 3379$}

For this galaxy we have additional data available and have used these to do two additional tests. The first relates to the possible influence of a color gradient (0.3 mag measured between 2 arcsec and 120 arcsec), the existence of which implies that the luminous mass distributions based on the $B$-band surface brightness profile will be slightly 


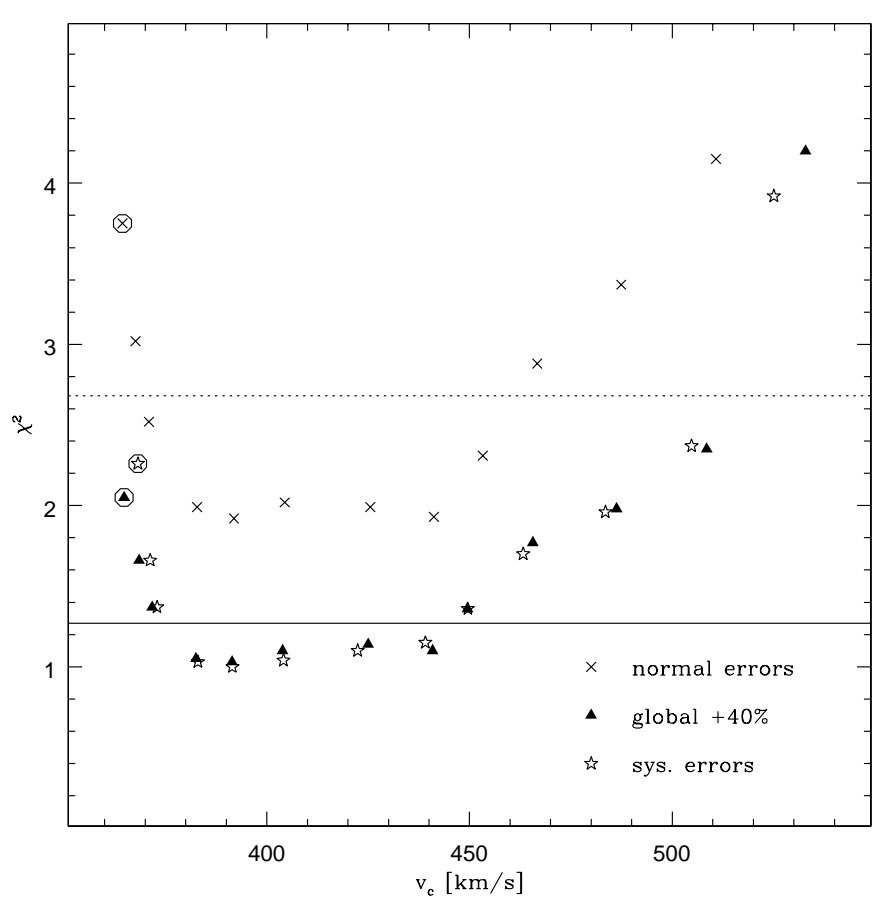

Fig. 22. $\chi^{2}$ results for NGC 1399, testing the two methods of accounting for systematic errors in the kinematic data. Crosses show $\chi^{2}$ results from fitting a set of models near the best-fitting region to the original errors shown in $\mathrm{S}+2000$. The dotted line shows the corresponding rescaled $95 \%$ confidence line according to Sect. 4 . Filled triangles are the $\chi^{2}$ values obtained when fitting the models to the data with errors globally increased by $40 \%$. The open stars result from fitting the models to the data after adding systematic "floors" to the error bars according to Table 5 . The full line shows the $95 \%$ confidence line corresponding to these values of $\chi^{2}$, again from Monte Carlo simulations. The range of allowed models is almost independent of which method is used, and the self-consistent model (circled) is ruled out in all three cases

different from that based on the $R$-band profile. To see how much this changes the dynamical models and the inferred circular velocity curves we have done the analysis separately for both bands. Therefore Figs. 10, 12, 18 and 20 each contain two panels for NGC 3379, referring to the $B$-Band and $R$-band models, respectively. From these plots it can be seen that the implied differences in the fits to the kinematic data, the intrinsic velocity anisotropy, and the circular velocity curves are small.

Secondly, independent kinematic data along four slit directions are available from Statler \& Smecker-Hane (1999). The corresponding velocity dispersion and $h_{4}$ profiles differ slightly from each other, possibly indicating a weakly triaxial shape of the galaxy. To see how this might influence our model results, we have done the analysis independently for all of these position angles. Figure 23 shows the four fits to their data in comparison with the fit derived earlier for the data shown in Sect. 2. The data taken from Statler \& Smecker-Hane (1999) have larger

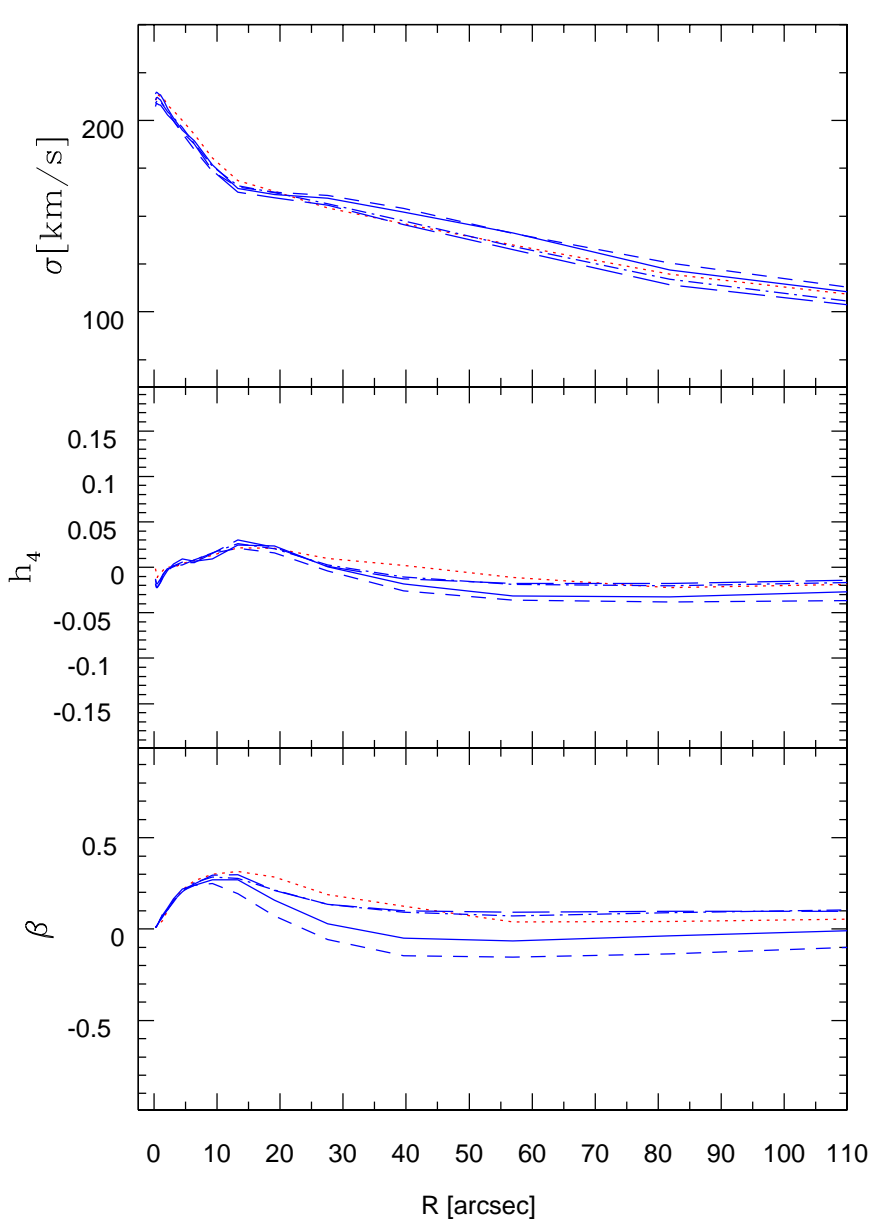

Fig. 23. Comparison of the best-fit models for NGC 3379 obtained from our data (solid line) and that from Statler \& Smecker-Hane (1999) along several slits (dotted, dashed, dotdashed and long-dashed lines for major axis, minor axis, $\mathrm{PA}=25$, and $\mathrm{PA}=115$, respectively). Top panel: Velocity dispersion versus radius. Middle panel: $h_{4}$ profile. Bottom panel: the inferred velocity anisotropy $\beta$

error bars and can be fitted with smaller $\chi^{2}$ than our data, which result in $\chi^{2}=3.205$ due to large point-topoint variations. Nonetheless all models for the five data sets show good agreement in the fits to $\sigma$ and $h_{4}$, and even in the more sensitive intrinsic anisotropy parameter $\beta$ the differences are only $\sim \pm 0.1$. The resulting cumulative mass and $M / L$ curves agree to better than five percent.

In addition, radial velocity measurements for $29 \mathrm{PNe}$ are available for NGC 3379 (Ciardullo et al. 1993). The maximum likelihood analysis (using the $B$-band models) shows that the SC model is consistent with the data as already noted by Ciardullo et al. (1993). However, since the PNe sample is very small the likelihood analysis does not constrain the range of the valid models strongly. Thus models with circular velocities in the range of $240 \mathrm{~km} \mathrm{~s}^{-1}$ to $350 \mathrm{~km} \mathrm{~s}^{-1}$ are within the $95 \%$ confidence interval; see Fig. 24. 


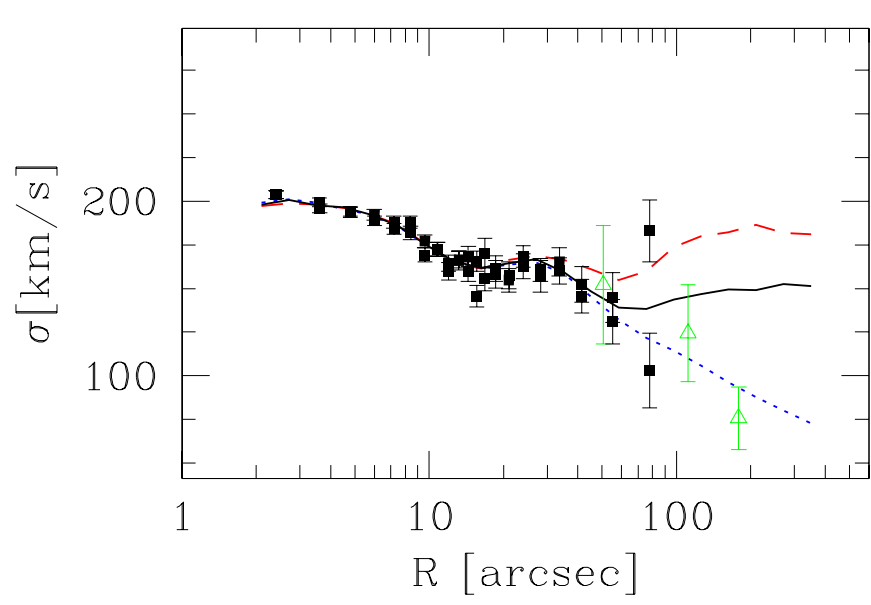

Fig. 24. The 29 PN of NGC 3379 binned (triangles) and plotted with the absorption line data (filled squares). The same three models as in Fig. 12 are plotted

\section{6. $N G C 6703$}

NGC 6703 was analysed by $\mathrm{G}+98$ as a prototype for the analysis method used here. They made the simplifying assumption of using a Jaffe-model for the photometry, with $R_{\mathrm{J}}=46.5 \operatorname{arcsec}$ and $L_{\mathrm{B}}=4.1610^{10} h_{50}^{-2} L_{\odot, B}$, and employed a set of 20 basis functions in the analysis. They found that potentials with $v_{\mathrm{c}}=250 \pm 40 \mathrm{~km} \mathrm{~s}^{-1}$ at the outermost kinematic data point fitted the data well in a $\chi^{2}$-sense and the SC-model was ruled out by a large margin. We redid the analysis using the same kinematic data, but inverting the SB-profile as described in Sect. 4 and using the extended basis set of 59 DFs as for the other galaxies of the present sample. The new results are shown in Figs. 10, 12, 18 and 20 and supersede those of G+98.

Comparison with the older results shows the following. The agreement of the deprojected density profile with the Jaffe profile is very good except for the very inner parts and a few small bumps round $R_{\mathrm{e}}$ also seen in the SB profile. Nevertheless the new models employing the extended basis tend to favor smaller $v_{\mathrm{c}}$ by $20-30 \mathrm{~km} \mathrm{~s}^{-1}$, and the SC model, although near the lower boundary of the confidence intervall, fits the data well in a $\chi^{2}$ sense, even though it still lies below many of the $h_{4}$ data points at intermediate radii. To test the origin of this difference we have also computed models with the new basis and smoothing parameter $\lambda$ but for the old Jaffe density profile. This showed that the greater part of the change is due to the larger basis used here. Apparently the smaller basis still implied residual smoothing through the basis functions, which is not negligible given the significant amount of substructure seen in the NGC 6703 kinematic profiles (in particular, the sharp dip in $h_{4}$ near $5^{\prime \prime}$, and the structure near $30^{\prime \prime}$ in $\sigma$ ), and thus influences the $\chi^{2}$-analysis. The model profiles on which the tests of $\mathrm{G}+98$ were carried out, and many of the other galaxies analysed here do not have such sharp substructures. The shape of the circular velocity curve, the anisotropy profile, and the stellar mass-to-light ratio are not strongly affected (note that the value given in Table 7 is for a different distance than the values given by $\mathrm{G}+98$ ).

\section{7. $N G C 7145$}

The drop in the velocity dispersion inside of $R=10$ arcsec possibly indicates the presence of central disk seen faceon. Although it is possible to produce a satisfying fit to the kinematics, the resulting rise in $M / L$ could be due not to the dark matter halo but to the transition from the disk-dominated to a random motion-dominated region.

\section{8. $N G C 7626$}

For this galaxy we again used both methods described in Sect. 4 for deriving $\chi^{2}$ values when the scatter in the data exceeds the observational errors. The results from the second method were marginally better than those obtained with globally increasing the error bars, and they are therefore shown in the figures.

\section{9. $N G C 3193$}

The BSG data for this galaxy have two very high velocity dispersion points with large errors on one side of the galaxy at $R \gtrsim 20^{\prime \prime}$. For the modelling we have assumed that there is some problem with these points, and have used only the data points on the other side with significantly lower dispersions and smaller errors.

\subsection{NGC 3640}

The outermost pairs of points for $\sigma$ and $h_{4}$ in the original data deviate from each other by much more than their error bars. Thus the errors of these points were replaced by one half the separation (see Fig. 15). Even afterwards the fits yield values of $\chi^{2}$ larger than unity $\left(\chi^{2}=1.7\right.$ for the best fitting model). Thus the confidence interval was determined by increasing the errors by $30 \%$.

\subsection{1. $N G C 4278$}

For this galaxy, Bertola et al. (1993) estimated the massto-light ratio from the kinematics of an extended gas ring. Converted to the distance used in the present paper, they found values for the $M / L_{\mathrm{B}}$ of 2.9 at $0.5 R_{\mathrm{e}}$ and 33 at $10 R_{\mathrm{e}}$. The corresponding values at these radii of our "best" model are 9.6 and 19.4, respectively, i.e., our profile is less steep than theirs. 


\subsection{2. $N G C 4472$}

We used the major axis data for the fit. BSG show data points out to $43^{\prime \prime}$. From the spectra, two further velocity dispersion points on both sides of the nucleus could be obtained, extending the profile to $R \simeq 55^{\prime \prime}$. However, it was not possible to derive corresponding $h_{4}$ values. The data points within $R<3$ arcsec were not fitted. The increase of $\sigma$ and $h_{4}$ towards the center may be the kinematic signature of a central dark mass.

Sharples et al. (1998) provide a list of $57 \mathrm{GC}$ velocities for NGC 4472. The maximum likelihood analysis result is that the GC-data allow a range of $v_{\mathrm{c}}=400-660 \mathrm{~km} \mathrm{~s}^{-1}$ and thus do not constrain the potentials as strongly as the absorption-line data do, again because of the small number of velocities. To compare the data "by eye" with our models we calculated the GC velocity dispersion for 5 bins containing 10 and 17 GCs. The same was done for 8 satellite galaxies of NGC 4472 taken from Binggeli (1993) of which 5 are believed to be "real" and 3 "possible" satellites. Figure 25 shows the combined velocity dispersion data together with "best" and boundary models for the absorption line data. The dispersion for the satellite galaxies should be considered as an upper limit. A model with a somewhat less massive halo than included in the "best" model, but well within the allowed range, would fit the combined data well.

In Fig. 26 we plot the X-ray mass-profile from Schindler et al. (1999) over our models. The "best" model from the absorption line kinematics provides a very good match to the $\mathrm{X}$-ray mass profile at large radii. Here, the significant result is the agreement in the normalisation. The functional form assumed for our halo models implies an outer mass profile linearly increasing with the radial distance. In the case of NGC 4472 (but see NGC 1399, $\mathrm{S}+2000$, and NGC 4636 below) this is similar to the radial profile derived from the X-ray emission under the assumption of isothermality.

\subsection{3. $N G C 4486$}

For NGC 4486 there is a large sample of GC velocities compiled by Cohen \& Ryzhov (1997) and Cohen (1999), containing 224 clusters. The maximum likelihood analysis for this sample shows that a range of potentials corresponding to a circular velocity between $490 \mathrm{~km} \mathrm{~s}^{-1}$ and $600 \mathrm{~km} \mathrm{~s}^{-1}$ at $R=42$ arcsec, the outermost stellarkinematic data point, is consistent with the GC velocity data. As noted by Kissler-Patig \& Gebhardt (1998) the outer GCs of NGC 4486 show considerable rotation $\left(\bar{v}=377.76\right.$ versus $\sigma=438.20 \mathrm{~km} \mathrm{~s}^{-1}$ for the outermost bin with $R>400$ arcsec). At smaller radii, $v / \sigma \leq 0.2$, however. If we exclude the GCs outside 400 arcsec, the range of valid potentials is narrowed to $490-570 \mathrm{~km} \mathrm{~s}^{-1}$.

As for NGC 4472 we binned the data and calculated the velocity dispersion (bin-size 20, 24 for the outermost

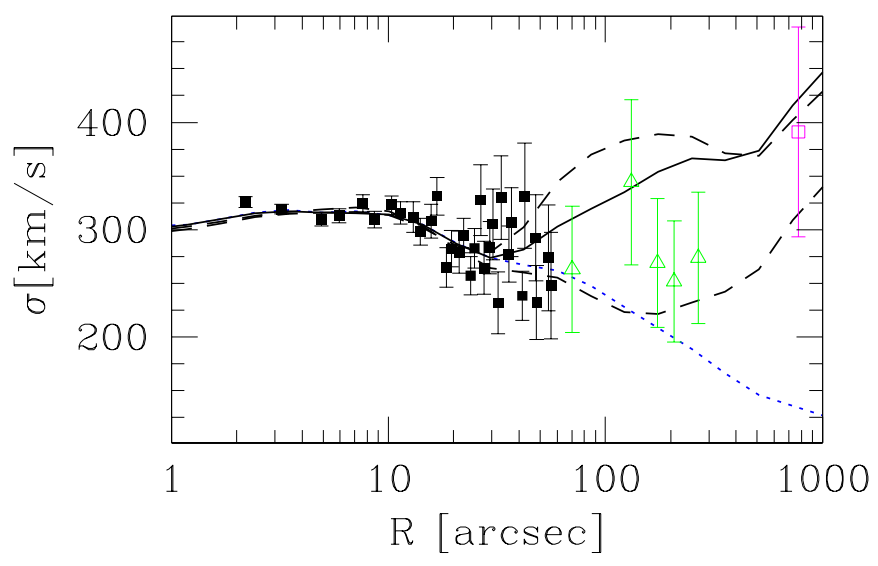

Fig. 25. GC (triangles) and dwarf galaxy (open square) velocity dispersions in NGC 4472 plotted over the stellar kinematic velocity dispersions (solid squares) and corresponding dynamical models. Solid line: "best" model; dashed lines: models at the boundaries of the confidence interval; dotted line: SC model. Note that the dwarf galaxies mark an upper limit since they contain three candidates with high line-of-sight velocities which are possibly not satellites of NGC 4472

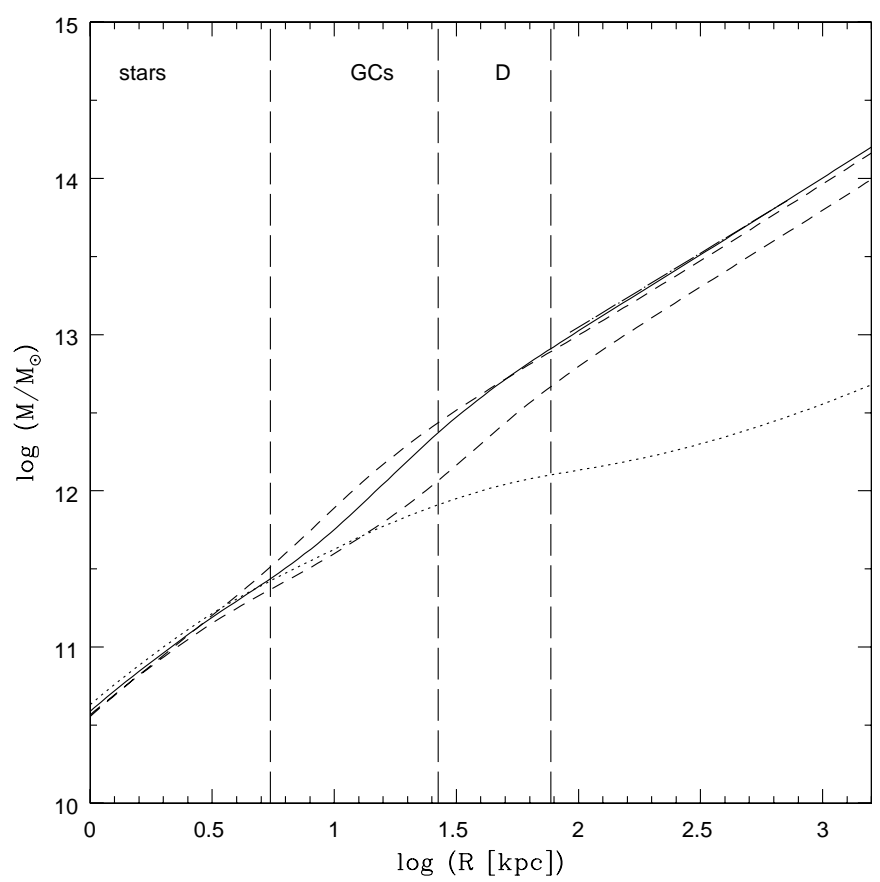

Fig. 26. Mass profiles for NGC 4472 derived from ROSAT $\mathrm{X}$-ray measurements for an isothermal gas (long dashed-dotted curve) compared to the models plotted in Figs. 25 and 16. The solid line is again the "best" model and provides a very good match to the X-ray mass profile at large radii. The dotted line is the constant mass-to-light model. The vertical line to the right of "stars" denotes the outer boundary of the stellar absorption line data, the one to the right of "GCs" the corresponding limit of the globular cluster data, and the line next to the "D" marks the mean position of the dwarf galaxies 


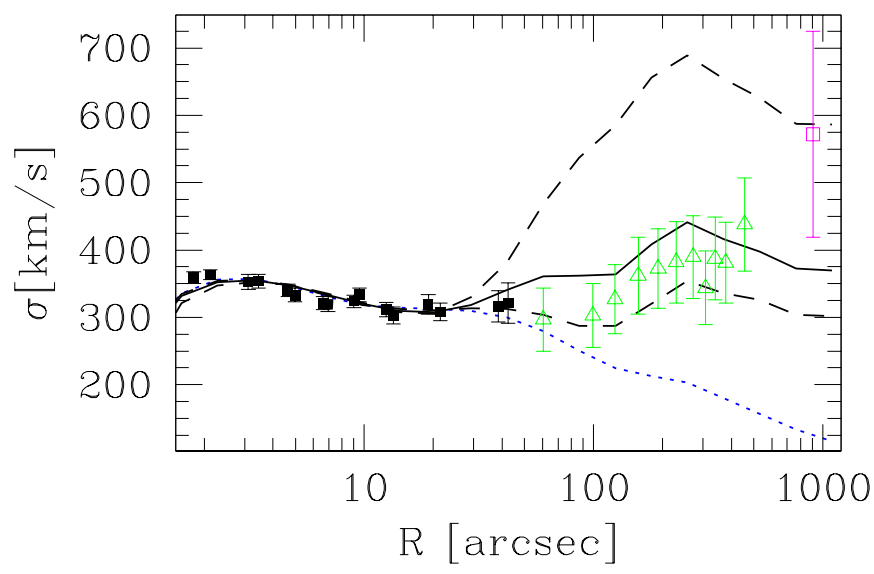

Fig. 27. GC (triangles) and dwarf galaxy (open square) velocity dispersions in NGC 4486 plotted over the stellar kinematics and the corresponding dynamical models. Solid line: "best" model; dashed lines: models at the boundaries of the confidence interval; dotted line: SC model. Note that the dwarf galaxies mark an upper limit since they contain three candidates with high line-of-sight velocities which may not be bound to NGC 4486

bin) for the GCs and for 8 satellite galaxies from Binggeli (1993). Also in this case 5 of the galaxies are bound to NGC 4486 and 3 are uncertain; thus the dispersion point for the galaxies is again an upper limit. In Fig. 27 we show the combined data together with the models derived from the stellar kinematic data. The data points fit perfectly into the confidence interval. Note that the SC model fails to match the GC data whereas it is within the confidence interval set by the absorption-line data.

In Fig. 28 we plot the X-ray mass-profile from Schindler et al. (1999) over our models. Their profile was calculated under the assumption of an isothermal gas distribution. The "best" model from the absorption line kinematics provides a very good match to the X-ray mass profile at large radii. Like in NGC 4472 the significant part of this good match is the agreement in the normalisation.

\subsection{NGC 4486B}

For this galaxy we combined the major and minor axis data of BSG94. The errors for the pairs of velocity dispersion data points at 2 and 3.5 arcsec were set to onehalf their respective separations. The reconstructed model DFs have large rms deviations indicating that the models constructed for this galaxy are the least reliable in the sample.

\subsection{NGC 4494}

Using the value of the smoothing parameter determined by $\mathrm{MC}$ simulations results in $\chi^{2}=2.49$. The main contri-

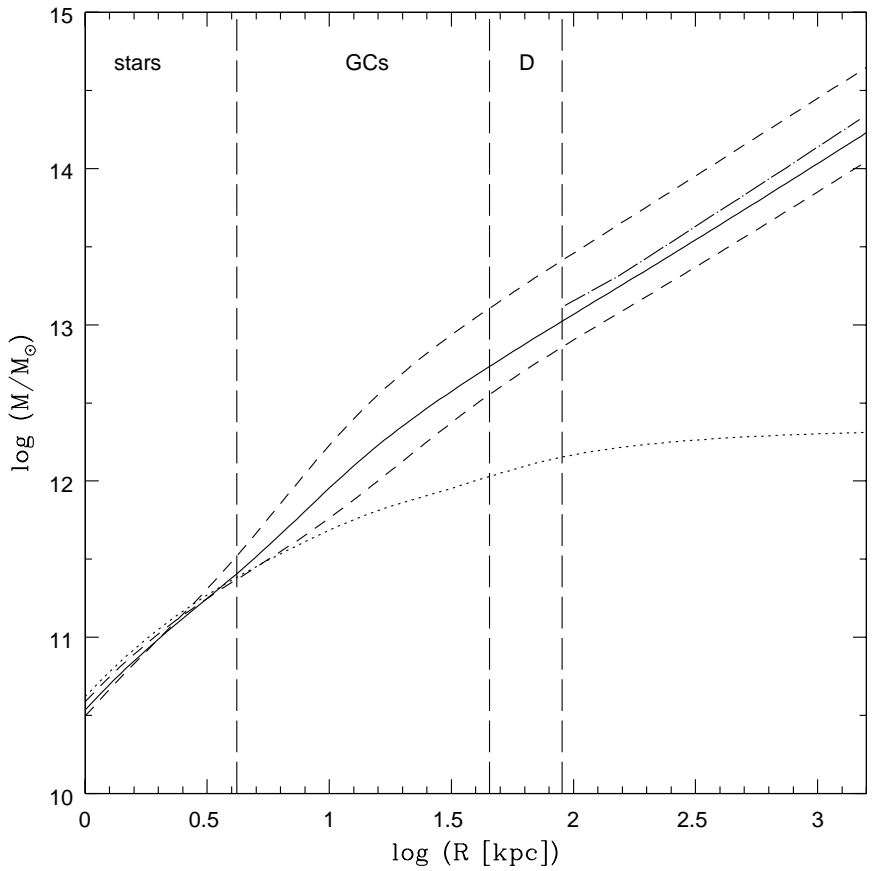

Fig. 28. Mass profiles for NGC 4486 derived from ROSAT $\mathrm{X}$-ray measurements for an isothermal gas (long dashed-dotted curve) compared to the models plotted in Figs. 27 and 16. The solid line is again the "best" model and provides a very good match to the X-ray mass profile at large radii. The dotted line is the constant mass-to-light model. The vertical lines have the same meaning as in Fig. 26

bution to this large $\chi^{2}$ stems from the large point-to-point variations in $\sigma$ between 5 and 10 arcsec, compared to the quoted errors. Increasing the errors by $60 \%$ allows the estimation of a confidence interval.

\subsection{6. $N G C 4636$}

The kinematic data have rather large errors. Both of the $h_{4}$ data points at 32 arcsec are probably spurious. Thus their errors were set to unity to ensure they do not influence the fit.

For NGC 4636 mass-profiles derived from ASCA X-ray data are available from Matsushita et al. (1998). Even in this case where the stellar kinematical models are not as extended and not as reliable as for the previous two galaxies with $\mathrm{X}$-ray data, the agreement is still reasonably good.

\section{Conclusions}

We have presented the results of a homogeneous dynamical analysis of a sample of 21 round galaxies (mostly E0-E1, four E2). The analysis is based on new extended kinematic data for eight ellipticals, reaching radii between 


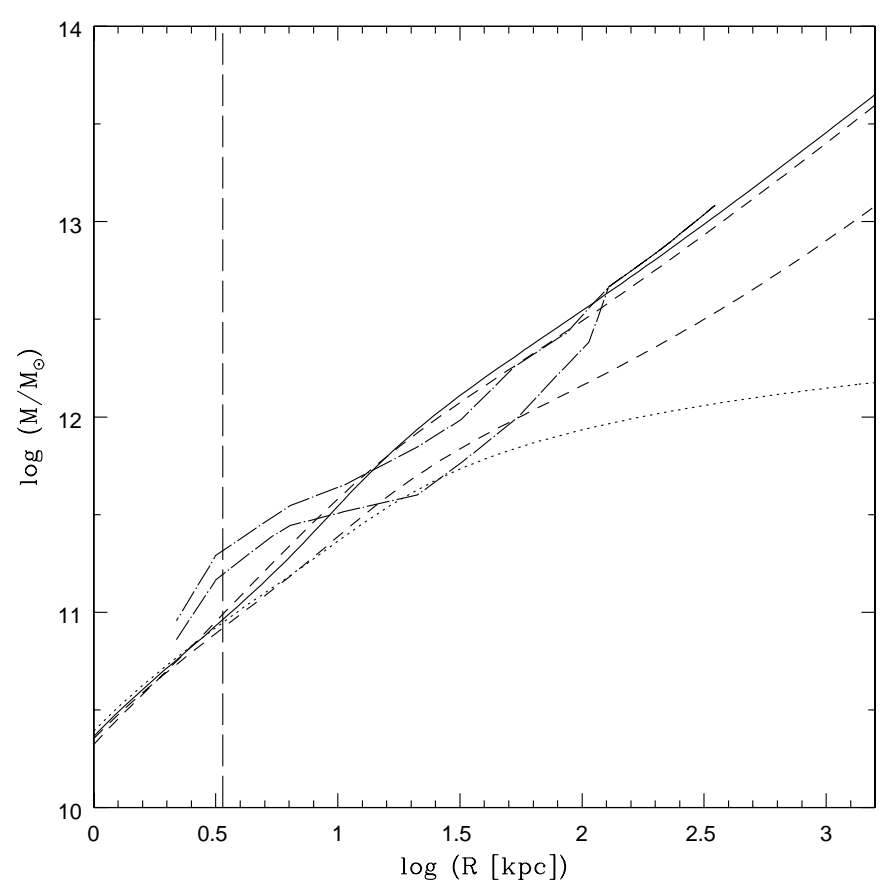

Fig. 29. Comparison of the ASCA X-ray mass-profile with the stellar kinematic mass models for NGC 4636. The vertical line marks the outer boundary of the stellar kinematics. The two long dashed-dotted lines denote the range of acceptable X-ray mass-profiles. Note the "bending" of the mass profile which was also observed for NGC 1399

$R_{\mathrm{e}}$ and $2 R_{\mathrm{e}}$, and data from the literature for the remaining galaxies. All these data include line profile information.

For each galaxy we have constructed a sequence of nonparametric spherical models in different luminous plus dark matter potentials. Comparing with the data we have determined a "best" potential model and a confidence range of models around it. From these we have determined circular velocity curves, mass-to-light ratio profiles, and anisotropy profiles for all galaxies, including error bands. We have done a variety of tests to investigate how the results are affected by details in the modelling and in how the data are treated, such as the choice of basis functions in the modelling (Sect. 5.3), treatment of systematic effects in the kinematic data (Sect. 4.2), colour gradients (Sect. 5.5), comparison of results from independent slit positions (Sect. 5.5), and small rotation (Sect. 5.1). From the statistics of apparent ellipticities the mean intrinsic shortto-long axis ratio for this sample of luminous ellipticals is 0.79 . We have concluded that the derived mass-to-light ratios will typically be underestimated by about $10 \%$ due to flattening of our galaxies along the line-of-sight, and that embedded face-on disks of the sizes known from near edge-on boxy ellipticals affect the anisotropy profiles only inside $\simeq 0.2 R_{\mathrm{e}}$ (Sect. 5.1). We have also cross-checked both the data and the modelling results with a variety of other work.
The sample includes seven galaxies with kinematic data extending to $R>1.5 R_{\mathrm{e}}$. Three of these show clear evidence for additional dark mass at these radii, in the sense that models based on luminous matter only are ruled out with $95 \%$ confidence: NGC 2434, NGC 7507, and NGC 7626. One, NGC 1399, shows weak stellar-dynamical evidence for dark matter, two others, NGC 3379, NGC 6703 are consistent with constant $M / L$ (revised analysis for NGC 6703). NGC 7145 is unclear because of a probable inner face-on disk. In addition, the less extended kinematics of NGC 315 also shows clear evidence for a radial increase in $M / L$.

Most of the galaxies with mass tracers other than stellar kinematics at larger radii (PNe, GCs, X-rays) also show evidence for dark matter: NGC 1399, 4472, 4486, 4636. In these cases the extrapolated dynamical models based on the stellar kinematic data match the independent models of the outer data very well. An exceptional case is NGC 3379, where also the outer PNe velocities are consistent with a constant $M / L$ model.

For all galaxies, we determine improved mass-to-light ratios. Generally, the respective "best" models show an outward increase of $M / L$. Although in many cases the data do not reach far enough to make this statistically significant, the agreement with additional outer data in the few cases cited above suggests that this may often be real.

The circular velocity curves (CVCs) resulting from our model fits are all consistent with being flat outside $R / R_{\mathrm{e}} \sim 0.3$ at the $\sim 10 \%$ level. Extended flat rotation curves, however, can only be demonstrated for about eight of the galaxies. In some galaxies, e.g., NGC 1399, 3379, the CVC rises to a maximum at $R / R_{\mathrm{e}} \sim 0.3 \sim 10^{\prime \prime}$, before falling by about $10 \%$ to reach the flat part at $R / R_{\mathrm{e}} \sim 1$. In others, e.g., NGC 2434, 7626, no such maximum is seen before the flat part of the CVC is reached.

The "best" models for most galaxies are isotropic to slightly radially anisotropic, with typical $\beta \simeq 0.3$, in a few cases $\beta \lesssim 0.5$ at $R_{\mathrm{e}} / 2$. Many then seem to turn back to nearly isotropic at larger radii. In their central regions, most of these galaxies also tend to become more isotropic. While the trend is significant, particularly in those galaxies where it starts at around $10^{\prime \prime}$, the detailed structure in the innermost few arcsec is not resolved by the data.

In summary, these results suggest a surprising degree of uniformity in the dynamical properties of elliptical galaxies, with the dark matter halo setting in at radii slightly larger than the effective radius.

Acknowledgements. The authors would like to thank M. Carollo and H.-W. Rix for providing the kinematic and photometric data for NGC 2434 in electronic form, M. Kissler-Patig for the list of GC data on his web-page, and T. Fischbacher and the physics department of the University of Munich for allowing us to use their Linux-cluster and for their help. It is a pleasure to thank B. Binggeli and D. Merritt for advice and the anonymous referee for his constructive comments. 
This work is partly based on observations carried out at the European Southern Observatory, La Silla, Chile. The authors thank the staff of the Calar Alto and ESO (in Garching and La Silla) observatories for their effective support. Some image reduction was done using the IRAF package distributed by the National Optical Astronomy Observatories. AK and OG were supported by grant 20-50676.97 from the Schweizerischer Nationalfonds, RPS and RB acknowledge the support by DFG grant SFB 375.

\section{References}

Arnaboldi M., Freeman K.C., Hui X., Capaccioli M., Ford H., 1994, The Messenger 76, 40

Balcells M., Carter D., 1993, A\&A 279, 376

Bender R., 1990, A\&A 229, 441

Bender R., Möllenhoff C., 1987, A\&A 177, 71

Bender R., Saglia R.P., Gerhard O., 1994, MNRAS 269, 785 (BSG94)

Bender R., Saglia R.P., 1999, ASP 182, 113

Bertin E., Arnouts S., 1996, A\&AS 117, 393

Bertin G., Bertola F., Buson L.M., et al., 1994, A\&A 292, 381

Bertola F., Pizzella A., Persic M., Salucci P., 1993, ApJ 416, L45

Bicknell G.V., Bruce T.E.G., Carter D., Killeen N.E.B., 1989, ApJ 336, 639

Binggeli B., 1993, Habilitationsschrift an der Universität Basel

Binney J.J., Tremaine S., 1987, Galactic Dynamics. Princeton Univ. Press

Burstein D., Davies R.L., Dressler A., et al., 1987 ApJS 64, 601

Caon N., Capaccioli M., Rampazzo R., 1990, A\&AS 86, 429

Caon N., Capaccioli M., D’Onofrio M., 1994, A\&AS 106, 199

Carollo C.M., Danziger I.J., Buson L., 1993, MNRAS 265, 553

Carollo C.M., Danziger I.J., 1994, MNRAS 270, 523

Carollo C.M., de Zeeuw P.T., van der Marel R.P., et al., 1995, ApJ 441, L25

Carollo C.M., Danziger I.J., Rich R.M., Chen X., 1997, ApJ 491,545

Ciardullo R., Jacoby G.H., Dejonghe H.B., 1993, ApJ 414, 454

Cohen J.G., Ryzhov A., 1997, ApJ 486, 230

Cohen J.G., 1999, astro-ph/9909471

Colless M.M., Burstein D., Wegner G., et al., 1993, MNRAS 262,475

Cretton N., van den Bosch F.C., 1999, ApJ 514, 704

Dehnen W., Gerhard O.E., 1993, MNRAS 261, 311

Dehnen W., Gerhard O.E., 1994, MNRAS 268, 1019

Davies R.L., Birkinshaw M., 1988, ApJS 68, 409

Davis L.E., Cawson M., Davies R.L., Illingworth G., 1985, AJ 90, 169

de Vaucouleurs G., de Vaucouleurs A., Corwin H.G. Jr., et al., 1991, Third Reference Catalogue of Bright Galaxies (RC3)

Djorgovski S., 1985, Ph.D. Thesis

Emsellem E., Dejonghe H., Bacon R., 1999, MNRAS 303, 495

Faber S., Wegner G., Burstein D., et al., 1989, ApJS 69, 763

Franx M., Illingworth G.D., Heckmann T., 1989, ApJ 344, 613
Gebhardt K., et al., 2000, AJ (in press), astro-ph/9912026

Gerhard O.E., 1993, MNRAS 265, 213

Gerhard O.E., 1994, in Galactic Dynamics and N-body Simulations, Contopoulos G., Spyrou N.K., Vlahos L. (eds.), Lecture Notes in Physics 433. Springer, Heidelberg, p. 191

Gerhard O.E., Jeske G., Saglia R.P., Bender R., 1998, MNRAS 295, $197(\mathrm{G}+98)$

Goudfrooij P., Hansen L., Jorgensen H.E., et al., 1994, A\&AS 104, 179

Ikebe Y., Ezawa H., Fukazawa Y., et al., 1996, Nat 379, 427

Jedrzejewski R., Schechter P.L., 1989, AJ 98, 147

Jørgensen I., Franx M., Kjærgaard P., 1995, MNRAS 273, 1097

Kaeppeli A., 1999, Diploma Thesis, Univ. of Basel

Keeton C.R., Kochanek C.S., Falco E.E., 1998, ApJ 509, 561

Kent S., 1984, ApJS 56, 105

King I.R., 1978, ApJ 222, 1

Kissler-Patig M., Gebhardt K., 1998, AJ 116, 2237

Kissler-Patig M., Brodie J.P., Schroder L.L., et al., 1998, ApJ 115, 105

Lauberts A., Valentijn E.A., 1989, The Surface Photometry Catalogue of the ESO-Uppsala Galaxies. Garching bei München: European Southern Observatory

Lauer T., 1985, ApJ 292, 292

Lauer T., et al., 1995, AJ 110, 2622

Loewenstein M., White R.E. III, 1999, ApJ 518, 50

Macchetto F., Pastoriza M., Caon N., et al., 1996, A\&AS 120, 463

Magorrian S.J., 2000, in From the Early Universe to the Present, ASP 197, 205

Matsushita K., Makishima K., Ikebe Y., et al., 1998, ApJ 499, L13

Matthias M., Gerhard O.E., 1999, MNRAS 310, 879

Mehlert D., Saglia R.P., Bender R., Wegner G., 1998, A\&A 332,33

Merritt D., 1993, ApJ 413, 79

Michard R., 1985, A\&AS 59, 205

Peletier R.F., Davies R.L., Illingworth G.D., Davis L.E., Cawson M., 1990, AJ 100, 1091

Prugniel Ph., 1997 (in preparation)

Rix H.-W., de Zeeuw P.T., Cretton N., van der Marel R., Carollo C.M., 1997, ApJ 488, 702

Saglia R.P., Bertin G., Stiavelli M., 1992, ApJ 384, 433

Saglia R.P., Bertin G., Bertola F., et al., 1993, ApJ 403, 567

Saglia R.P., Kronawitter A., Gerhard O.E., Bender R., 2000, AJ 119, 153 (S+2000)

Schechter P.L., Gunn J.E., 1979, ApJ 229, 472

Schindler S., Binggeli B., Böhringer H., 1999, A\&A 343, 420

Sembach K.R., Tonry J.L, 1996, AJ 112, 797

Sharples R.M., Zepf S.E., Bridges T.J., et al., 1998, AJ 115, 2337

Statler T.S., Smecker-Hane T., 1999, AJ 117, 839

Tremblay B., Merritt D., 1996, AJ 111, 2243

van der Marel R.P., 1991, MNRAS 253, 710

Wahba G., Wendelberger J., 1980, Month. Weather Rev. 108, 1122

Zabludoff A.I., Mulchaey J.S., 1998, ApJ 496, 39 